\title{
A model of polarized-beam AGS in the ray-tracing code Zgoubi
}

\author{
F. Méot, L. Ahrens, K. Brown, Y. Dutheil, \\ J. Glenn, H. Huang, T. Roser, V. Schoefer, N. Tsoupas
}

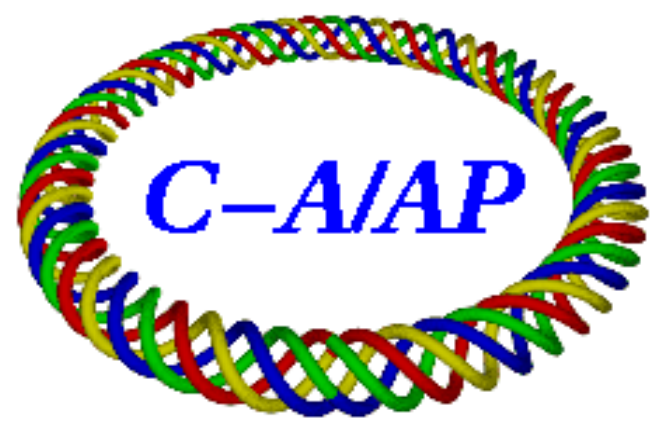

Collider-Accelerator Department Brookhaven National Laboratory Upton, NY 11973

\author{
U.S. Department of Energy \\ Office of Science, Office of Nuclear Physics
}

Notice: This document has been authorized by employees of Brookhaven Science Associates, LLC under Contract No. DE-SC0012704 with the U.S. Department of Energy. The United States Government retains a nonexclusive, paid-up, irrevocable, world-wide license to publish or reproduce the published form of this document, or allow others to do so, for United States Government purposes. 


\title{
A model of polarized-beam AGS in the ray-tracing code Zgoubi
}

\author{
F. Méot, L. Ahrens, K. Brown, Y. Dutheil, J. Glenn, H. Huang, T. Roser, V. Schoefer, N. Tsoupas \\ Collider-Accelerator Department, BNL, Upton, NY 11973
}

July 12, 2016

\begin{abstract}
A model of the Alternating Gradient Synchrotron, based on the AGS snapramps, has been developed in the stepwise ray-tracing code Zgoubi. It has been used over the past 5 years in a number of accelerator studies aimed at enhancing RHIC proton beam polarization. It is also used to study and optimize proton and Helion beam polarization in view of future RHIC and eRHIC programs. The AGS model in Zgoubi is operational on-line via three different applications, 'ZgoubiFromSnaprampCmd', 'AgsZgoubiModel' and 'AgsModelViewer', with the latter two essentially interfaces to the former which is the actual model 'engine'. All three commands are available from the controls system application launcher in the AGS 'StartUp' menu, or from eponymous commands on shell terminals. Main aspects of the model and of its operation are presented in this technical note, brief excerpts from various studies performed so far are given for illustration, means and methods entering in ZgoubiFromSnaprampCmd are developed further in appendix.
\end{abstract}

Tech. Note $C-A / A P / 566$

$B N L C-A D$ 


\section{Contents}

\section{INTRODUCTION}

2 AGS ZGOUBI MODEL COMMANDS

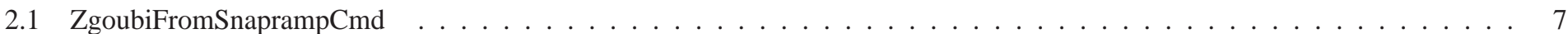

2.2 AgsZgoubiModel interface $\ldots \ldots \ldots \ldots \ldots \ldots \ldots \ldots$

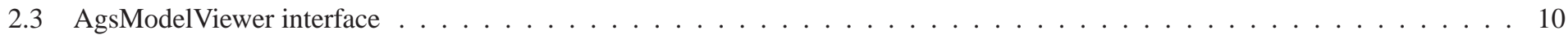

3 THE MODEL OF THE AGS IN Zgoubi 10

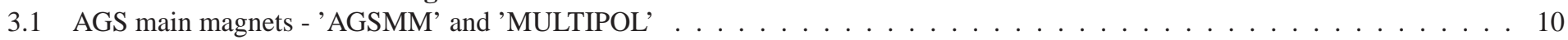

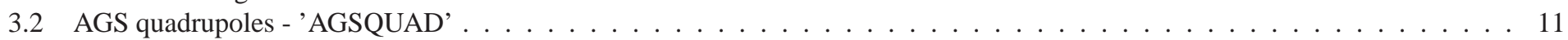

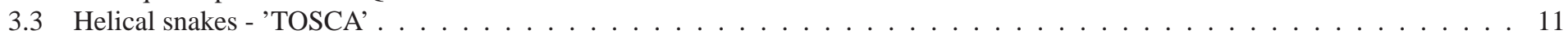

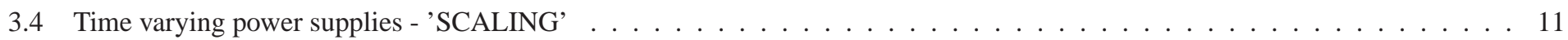

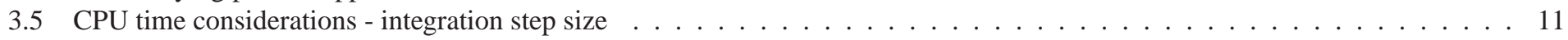

4 SAMPLE SIMULATIONS

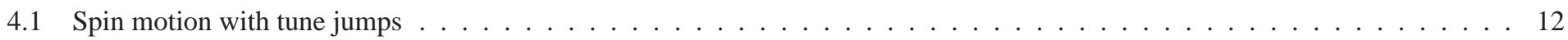

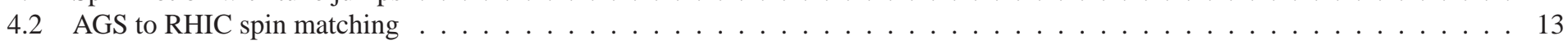

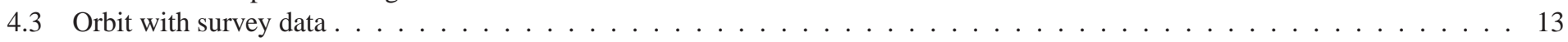

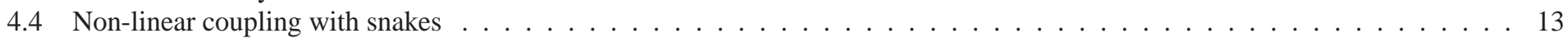

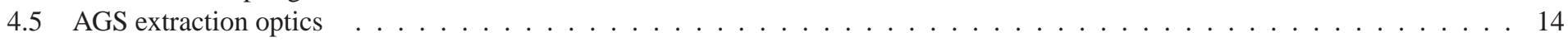

5 WHERE ARE THE FILES ? 16

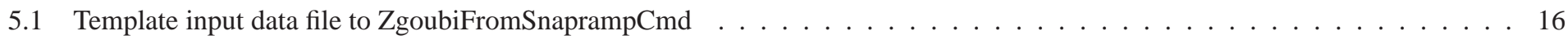

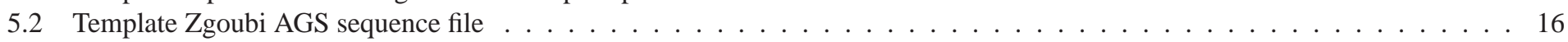

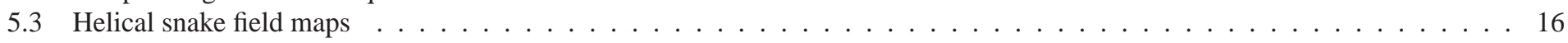

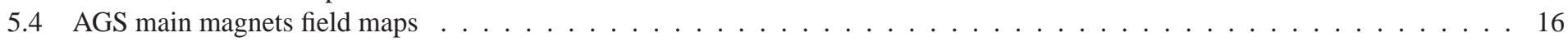

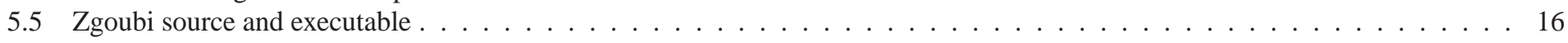

5.6 ZgoubiFromSnaprampCmd and ancillary source files $\ldots \ldots \ldots \ldots \ldots \ldots$

6 CONCLUSION

$\begin{array}{ll}\text { APPENDIX } & 18\end{array}$

A Zgoubi method, in short 18

B Model of the AGS in Zgoubi 18

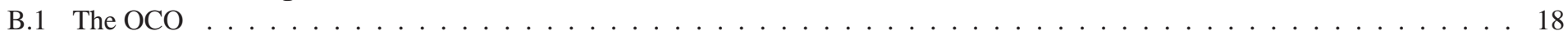

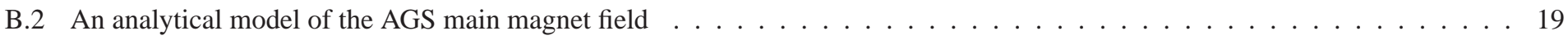

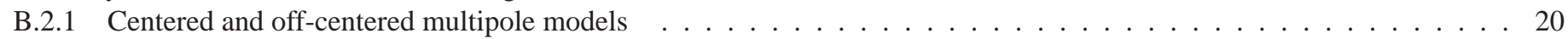

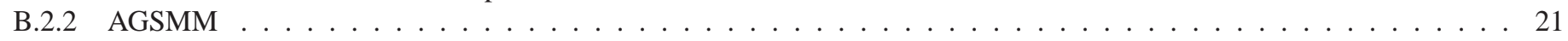

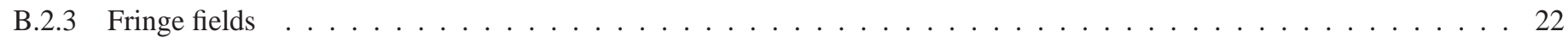

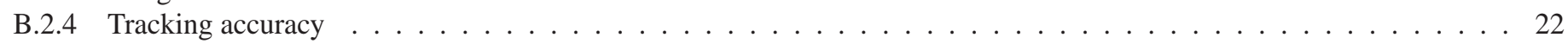

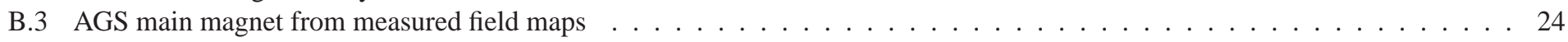

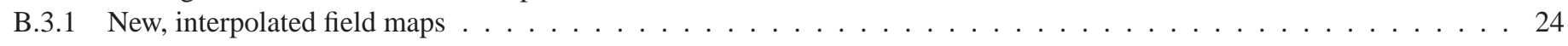

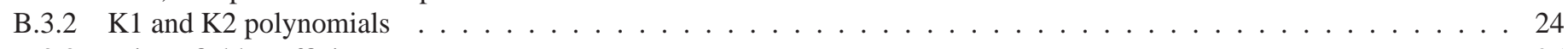

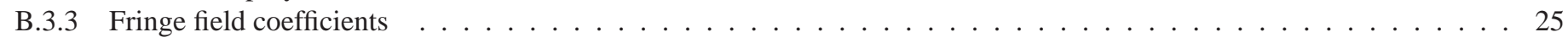

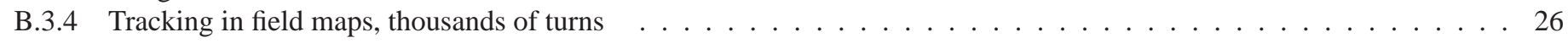

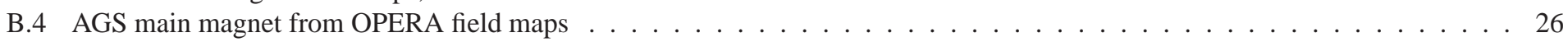

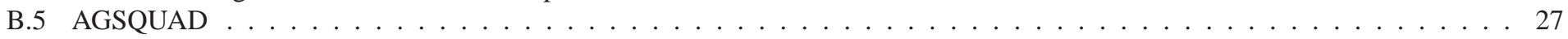

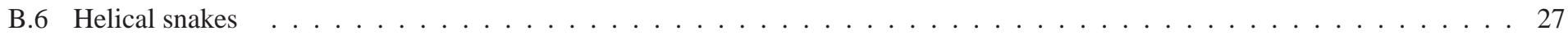

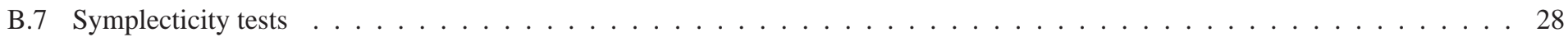

C From AGS snapramps to Zgoubi to the AGS model : functionning of ZgoubiFromSnaprampCmd 29

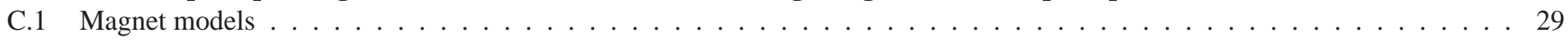

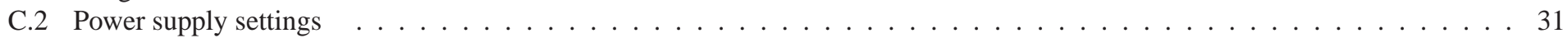

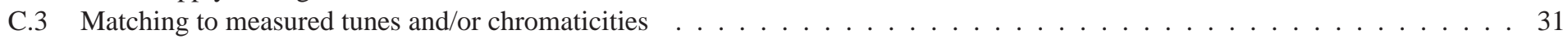


D I/O files to/from ZgoubiFromSnaprampCmd 32

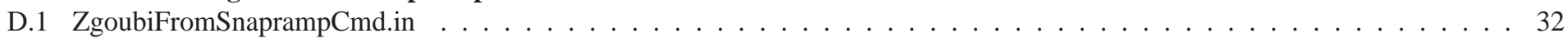

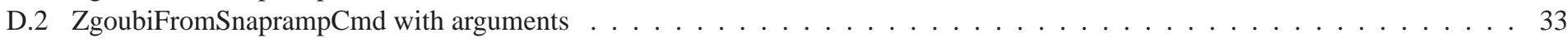

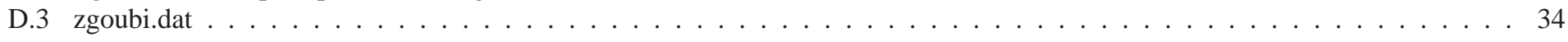

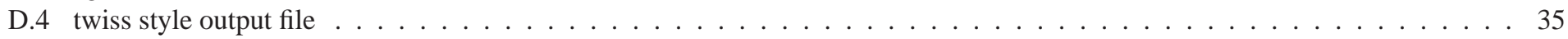




\section{INTRODUCTION}

This technical note is a working document, in addition to giving a status of the AGS model in the ray-tracing code Zgoubi (still far from a 'finish product') it is aimed at easing the use, and further development, of these software tools.

A model of the Alternating Gradient Synchrotron accounting for polarized beam lattice aspects has been developed over the past $5 \sim 6$ years, based on the stepwise ray-tracing code Zgoubi [1, 2] (see App. A). The development of this model started in 2009 2010 [3]-[11]. It strongly benefited from the 'AGS On-Line Model' meeting run since then at C-AD [9]. The resulting software tools have been extensively used in a number of studies regarding beam and polarization transport simulations, such as the assessment of machine and spin equipment settings for polarized proton RHIC runs [11], non-linear coupling effects induced by the helical snakes at low energy [8], studies and developments regarding the cold and warm snake field maps [9, 12], AGS instrumentation [13], polarization transport from AGS to RHIC injection kicker [14, 15], and more [11, 16].

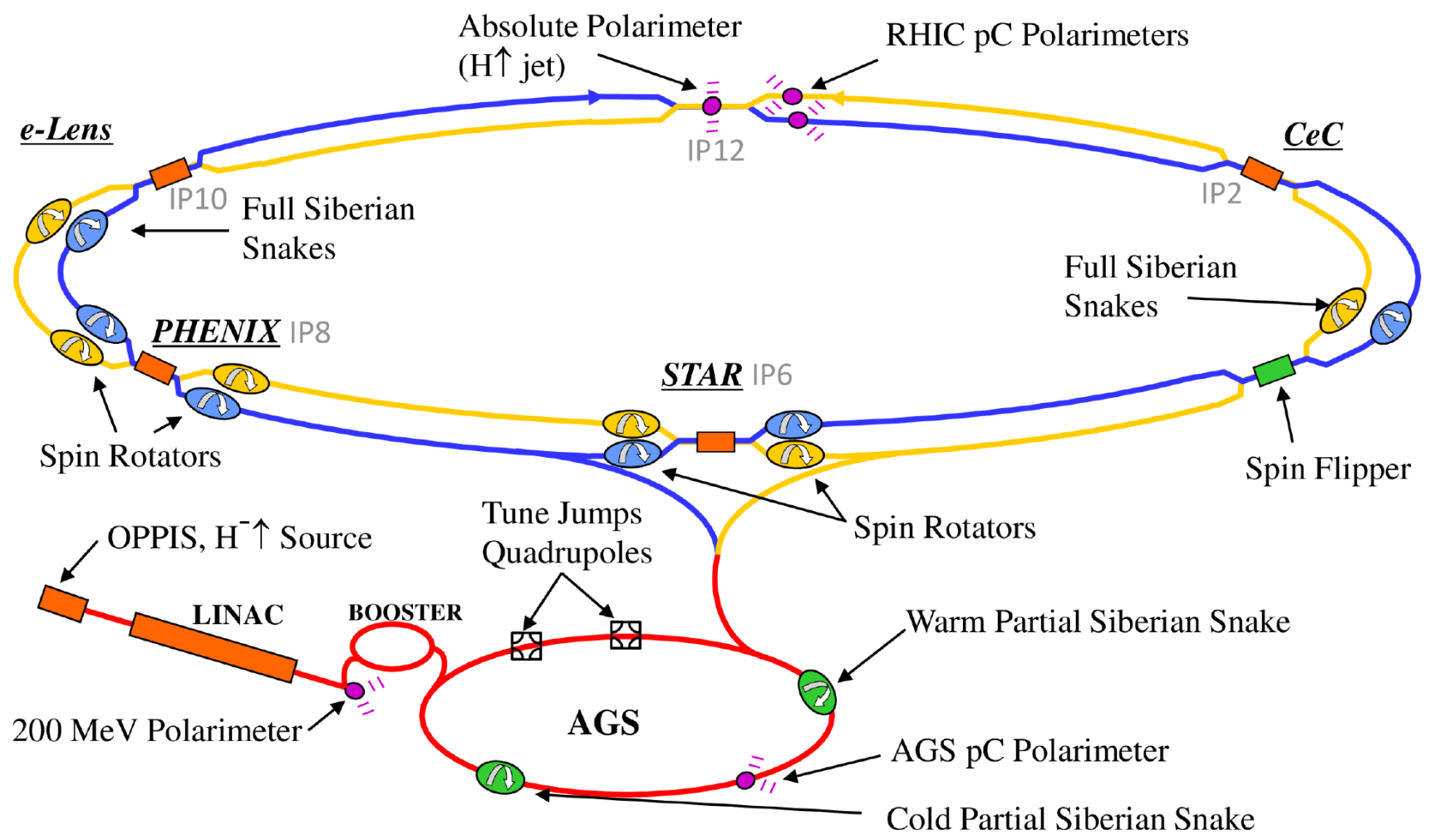

Figure 1: RHIC collider and its injector chain. In the AGS, the cold and warm partial snakes, as well as the two tune jump quadrupoles, are part of proton beam polarization control equipment.

There are several reasons for opting for stepwise integration methods, amongst the many possible ways of computing machine optics and tracking polarized particles, motivated in a first approach by the goal of accurate spin tracking simulations, in relation with the following :

- two strong helical dipoles ('partial snakes') are used in the AGS (Fig. 1) to overcome imperfection and vertical intrinsic depolarizing resonances [12]. These magnets perturb the optics at low energy (orbit, focusing, coupling, field non-linearities) [17], they are difficult to model analytically, regarding particle and spin transport,

- the AGS lattice is based on 240 combined function dipoles, these require good field modeling, featuring quadrupole and sextupole components, reproducing accurately their strength variation with magnet current (multipole components do not track one another), possibly reproducing actual fringe fields. Stepwise ray-tracing restitutes accurate trajectory geometries across these combined function magnets, in which the magnetic field is constantly varying along arcs of trajectories,

- the stepwise integration method allows realistic magnetic field models,

- especially, it allows using the OPERA field maps to represent the two AGS snakes,

- and it allows using measured or OPERA field maps in representing the various lattice magnets, for instance the 240 main dipoles.

In addition, stepwise ray-tracing had proven effective in various types of exercises, including earlier, similar simulation of spin tracking in a synchrotron [2] and other muon beam decay ring [18], highly non-linear ring optics [19], various studies in large rings [20, 21].

The ultimate goals in modeling the AGS are

(i) to provide an understanding of machine optics and spin dynamics,

(ii) to assess the effectiveness of, and provide guidance in developing, new polarization improvement methods.

The AGS model in Zgoubi can be operated both on-line and off-line, via three different applications, ZgoubiFromSnaprampCmd, AgsZgoubiModel and AgsModelViewer [22, 23]. The latter two are essentially interfacing programs to the former which is the actual model engine. To create a model of the ring, ZgoubiFromSnaprampCmd uses the AGS power supply (so-called) 'snapramp' data base. It works in a similar 
way to the MADX model command 'MadxFromSnaprampCmd' [24], from which actually those pieces of code that deal with conversion from snapramp currents to magnet fields have been drawn, for implementation in both ZgoubiFromSnaprampCmd and Zgoubi. All three commands are available from the controls system application launcher in the AGS 'StartUp' menu, or from the eponymous commands on shell terminals. All three allow producing a model of the live AGS, using the live snapramp, or working off-line, using archived snapramps.

ZgoubiFromSnaprampCmd can be executed in a shell terminal, and will produce models of the AGS at arbitrary (user specified) timings, or series of timings, along machine cycle. AgsZgoubiModel is mostly a research and development interface, used for development of the model in Zgoubi and its benchmarking against measured AGS data, it is essentially based on ZgoubiFromSnaprampCmd engine and on AGS Controls sdds data file treatment tools (regarding for instance tune, optical functions, chromaticity measurement records). AgsModelViewer is part of the AGS Controls tools, it also interfaces MadxFromSnaprampCmd, as well as the Booster model in MADX - note that a development of a Booster model in Zgoubi, based on snapramp records as well, has been undertaken recently in the context of future RHIC and eRHIC project physics programs [25]. AgsModelViewer provides a host of post-processing equipments and graphics. Requesting a model of the AGS using AgsModelViewer essentially results in an execution of ZgoubiFromSnaprampCmd engine at user specified timings. A model of the AGS at a particular timing takes the form of an input file to Zgoubi, ready for simulation of AGS optics at that particular timing, however, running ZgoubiFromSnaprampCmd will deliver in addition various Zgoubi I/O files which allow further computations as tune and/or chromaticity scans, optical functions, polarization parameters, start-to-end polarized bunch acceleration, etc.

This technical note is organized in the following way :

- Section 2 gives a brief description of ZgoubiFromSnaprampCmd, and of the two interfaces AgsZgoubiModel and AgsModelViewer,

- Section 3 addresses the very content of the engine : magnet models, use of field maps, various Zgoubi capabilities, etc.,

- Section 4 illustrates this with a series of examples of simulations,

- Section 5 informs on the location of zgoubi and ZgoubiFromSnaprampCmd source files, field map files, templates, etc., in C-AD computers.

Additional details regarding the elaboration of the model, possibilities of further developments and studies (such as using the 240 field maps of the main magnets, main magnet fringe fields, etc.) are discussed in a series of Appendices, as follows :

- App. A : a brief reminder regarding Zgoubi stepwise integration method,

- App. B : model of the AGS in Zgoubi, including OCO considerations ; analytical AGS magnet models ; helical snakes ; tracking accuracy considerations, etc.,

- App. C : from AGS snapramps to Zgoubi model : functionning of ZgoubiFromSnaprampCmd,

- App. D : I/O files to/from ZgoubiFromSnaprampCmd and zgoubi.

This technical note and the work that it describes lean on a host of earlier publications at BNL C-AD. A limited amount are cited, with a tendency to focus on publications more directly related to these AGS model developments, this is for the sake of efficiency. In the latter however, additional references to earlier works, by others, can be found. More material can be found in addition in BNL C-AD 'AGS On-Line Model' and 'Spin Meeting' series [9, 10], as well as in accelerator conference proceedings from 2010 on. 


\section{Table 1: How ZgoubiFromSnaprampCmd operates.}

ZgoubiFromSnaprampCmd is a standlone command, on shell terminals. It can also be interfaced using 'AgsZgoubiModel' (Sec. 2.2) or 'AgsModelViewer' (Sec. 2.3)

(i) ZgoubiFromSnaprampCmd reads magnet settings from an AGS snapramp (a folder that archives current evolution in all power supplies, over a machine cycle. All AGS cycle snapramps are archived in a dedicated AGS Controls data base).

(ii) ZgoubiFromSnaprampCmd then builds a (series of) 'zgoubi.dat' input data file(s) to Zgoubi at a particular (or series of) timing(s), one file per timing. These zgoubi.dat files are created using an existing template (App. 5.2, p. 16).

zgoubi.dat contains the AGS sequence, and appropriate commands for Zgoubi to execute (App. D.3, p. 34).

(iii) Each zgoubi.dat problem is then run, so producing various outputs, including a MADX style 'twiss' file, 'zgoubi.TWISS.out'. These ouput files are all archived in dedicated local folders:

- all zgoubi.TWISS.out in the same ./twiss folder,

- the various other output files in a './model.tttt.zgoubi' series, with 'tttt'=timing.

(iv) ZgoubiFromSnaprampCmd in addition executes various data treatment, following user's requests, such as tune computations, tune and/or chromaticity matching to measured AGS data, plots (as Figs. 2, 3), etc., found in ./model.tttt.zgoubi folders.

\section{Some more :}

- The informations needed by ZgoubiFromSnaprampCmd to operate, such as the snapramp to consider, timing(s), post-execution data treatment to be performed, etc., can be specified in two different ways :

- either as an input data file, ZgoubiFromSnaprampCmd.in (App. 5.1 and App. D.1),

- or as arguments to ZgoubiFromSnaprampCmd (App. D.2).

- Two templates zgoubi.dat files are available (App. 5.1) :

- one for polarized proton runs,

- one for ions.

They essentially differ by the former comprising cold and warm snakes. Which one to be used is part of the input information to ZgoubiFromSnaprampCmd.

- There also exists a template input data file ZgoubiFromSnaprampCmd.in (App. 5.1) : if ZgoubiFromSnaprampCmd is launched without arguments, from a folder that does not contain ZgoubiFromSnaprampCmd.in, then that template is used by default. 


\section{AGS ZGOUBI MODEL COMMANDS}

The following sections summarize what can be expected from the model and its two interfaces, and how.

\subsection{ZgoubiFromSnaprampCmd}

The model of the AGS in the ray-tracing code Zgoubi can be launched from a shell terminal under linux/unix, via the command ZgoubiFromSnaprampCmd. The structure of this command was inspired from the existing MadxFromSnaprampCmd, which provides a model of the AGS in MADX program [24]. The way ZgoubiFromSnaprampCmd operates is summarized in Tab. 1 (p. 6), more can be found in App. C (p. 29).

Running it produces a 'twiss' output file, formatted like MADX one (see App. D.4). This makes existing interfaces to MADX operational with both engines with minor additional work, which lead in particular to straightforward development of Zgoubi AGS model as a second engine in AgsModelViewer (Sec. 2.3). The twiss file contains such informations as the optical functions (orbits, betatron functions, phase advance, spin $\vec{n}_{0}$ vector, etc.) along the AGS.

Figs. 2 displays a scan so obtained, over a set of timings defined in ZgoubiFromSnaprampCmd.in :

- horizontal and vertical tunes, prior and next to matching Zgoubi model tunes to a measured AGS tune-scan,

- coupling strength and penalty value reached by the tune matching

- necessary main magnet $d K_{F, D} / K_{F, D}$ perturbations ( $d Q H_{I}, d Q V_{I}$ in main quadrupole families could be used instead) to have the model tunes agree with measured values (that method is discussed in App. C.3, p. 31).

Fig. 3 displays the residual orbits and the optical functions at extraction, including the extraction bumps at G10 and H10.

The model includes transition gamma quadrupoles (transition is at $\approx 310 \mathrm{~ms}$, Fig. 2), horizontal tune jump quadrupoles (their effect is apparent in Fig. 2, $\Delta Q_{x}=0.04$ at all $G \gamma \pm Q_{x}=$ integer, proton $\mathrm{G}=1.79284735, \gamma=$ Lorentz relativistic factor), as well as the G10 kicker and $\mathrm{H} 10$ septum extraction bumps (extending over $s \approx 400-550 \mathrm{~m}$, Fig. 3).

The AGS Zgoubi model which produced Figs. 2, 3 has been archived at

$$
\text { /rap/lattice_tools/zgoubi/AgsZgoubiModel/Ags_ZgoubiModel_examples/AGSOptics/Example_2015 - } 05 \text { - } 17
$$

comments can be found in the README file therein. It uses the AGS snapramp

$$
\text { /operations/app_store/RunData/run_fy15/fullRun/Ags/Snapramp/15Mar08/ppmUser4/15Mar08 - 0854_fgm_U4cur/ }
$$

Matching of model tunes uses measured tune scan

$$
\begin{aligned}
& \text { /operations/app_store/RunData/run_fy15/fullRun/Ags/TuneMeter/ } \\
& \text { TuneSCan_Sun_03_08_15_095118_150.000 - 610.000ms/ATM_DATA/ }
\end{aligned}
$$

\subsection{AgsZgoubiModel interface}

AgsZgoubiModel is an R\&D tool used for developing the AGS Zgoubi model. An example of interest is in the production of Fig. 2, as follows.

When producing a model of the AGS at particular timing(s) during a cycle, tunes (and chromaticities) will fall some distance from measured values. It may be necessary, for spin dynamics studies for instance, to have tight agreement between the two. This is handled by matching, with variables for instance K1F and K1D gradient indices in respectively focusing and defocusing main magnet families (and K2F, K2D for chromaticities), whereas the constraints are measured data. If such matching is requested, AgsZgoubiModel just requires the address of an sdds data file and will then establish the appropriate links, it will create a model using ZgoubiFromSnaprampCmd, and will launch the matching procedure. This will result in a (series of) zgoubi.dat matched AGS models, ready for further use.

Similar automatic procedures are under development in AgsZgoubiModel, for orbit, dispersion, including interfacing with the automatic dispersion acquisition application [26]. There are plans as well to link AgsZgoubiModel with the AGS Controls automatic chromaticity measurement procedure. AgsZgoubiModel on the other hand provides a convenient environment for developing and testing such new procedures prior to installation in AgsModelViewer (next section). 
ZgoubifromSnaprampCmd. AGS TUNES (after FIT to tune scan)

snapramp: .../run $\mathrm{f}$ 15/fullRun/Ags/Snapramp/15Mar08/ppmUser4/15Mar08-0854fgm 4 cur/

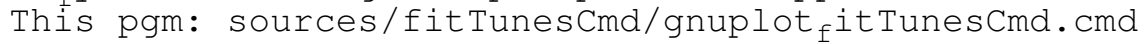

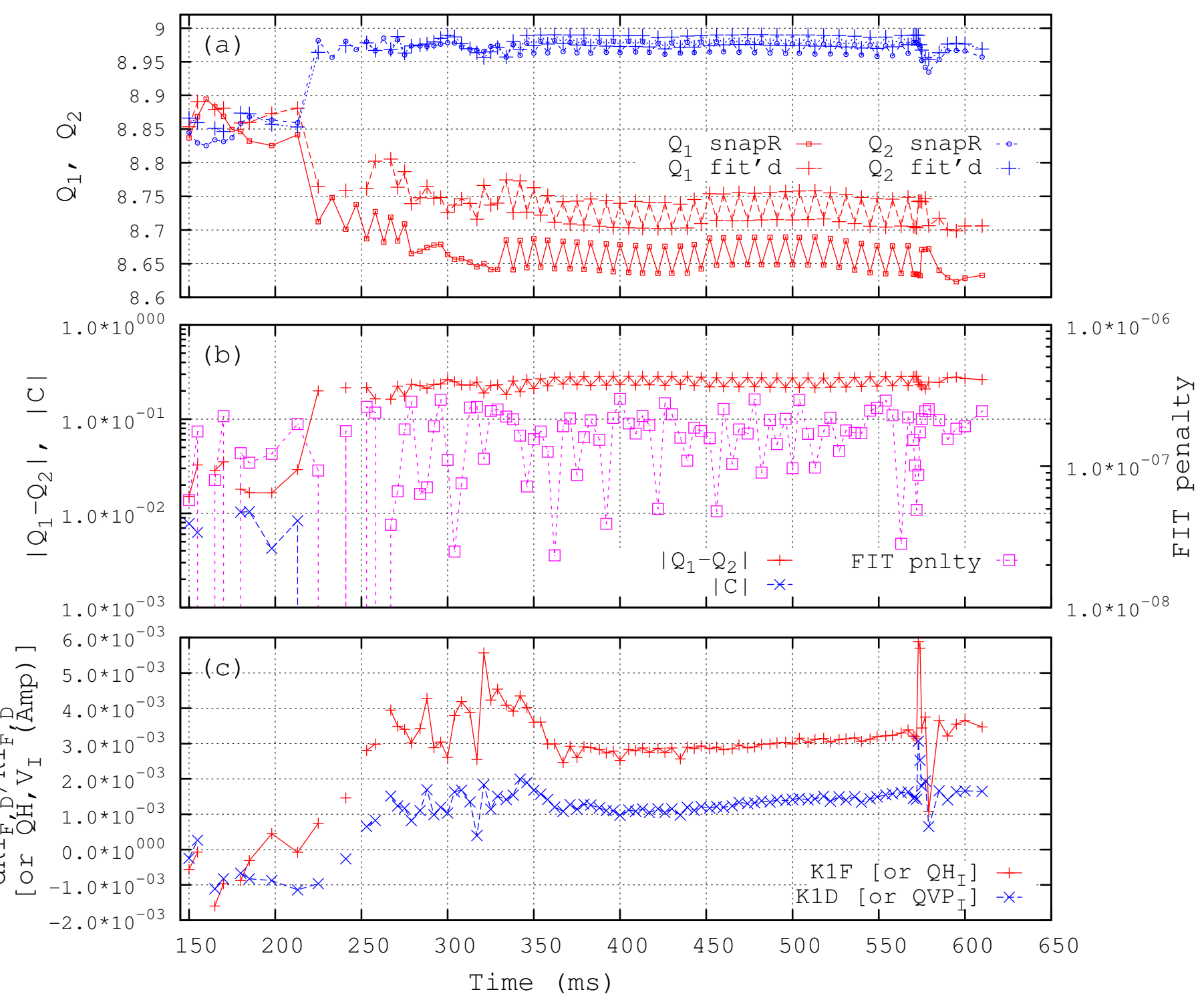

Figure 2: Top : evolution of the (coupled) tunes along an acceleration ramp, either prior to ('snapR' curves), or after ('fit'd' curves) the K1F and K1D indices of respectively the focusing and defocusing main dipole families have been adjusted for the model to match measured AGS tunes. Tune jump mode is apparent here, it uses tune jump quadrupoles Qjump_I05 and Qjump_J05 located in AGS 'I' and 'J' superperiods (see Fig. 1). Middle, left vertical axis : coupling strength and tune distance (after tune matching); right vertical axis : penalty values reached in the tune matching process. Bottom : the relative amount that main magnet $\mathrm{K} 1 \mathrm{~F}$ and $\mathrm{K} 1 \mathrm{D}$ need be varied in order to have measured and model tunes agree $\left(Q H_{I}\right.$ and $Q V P_{I}$ stand for the fact that main quadrupole current settings can be used for that purposed, instead, depending on user's request). 


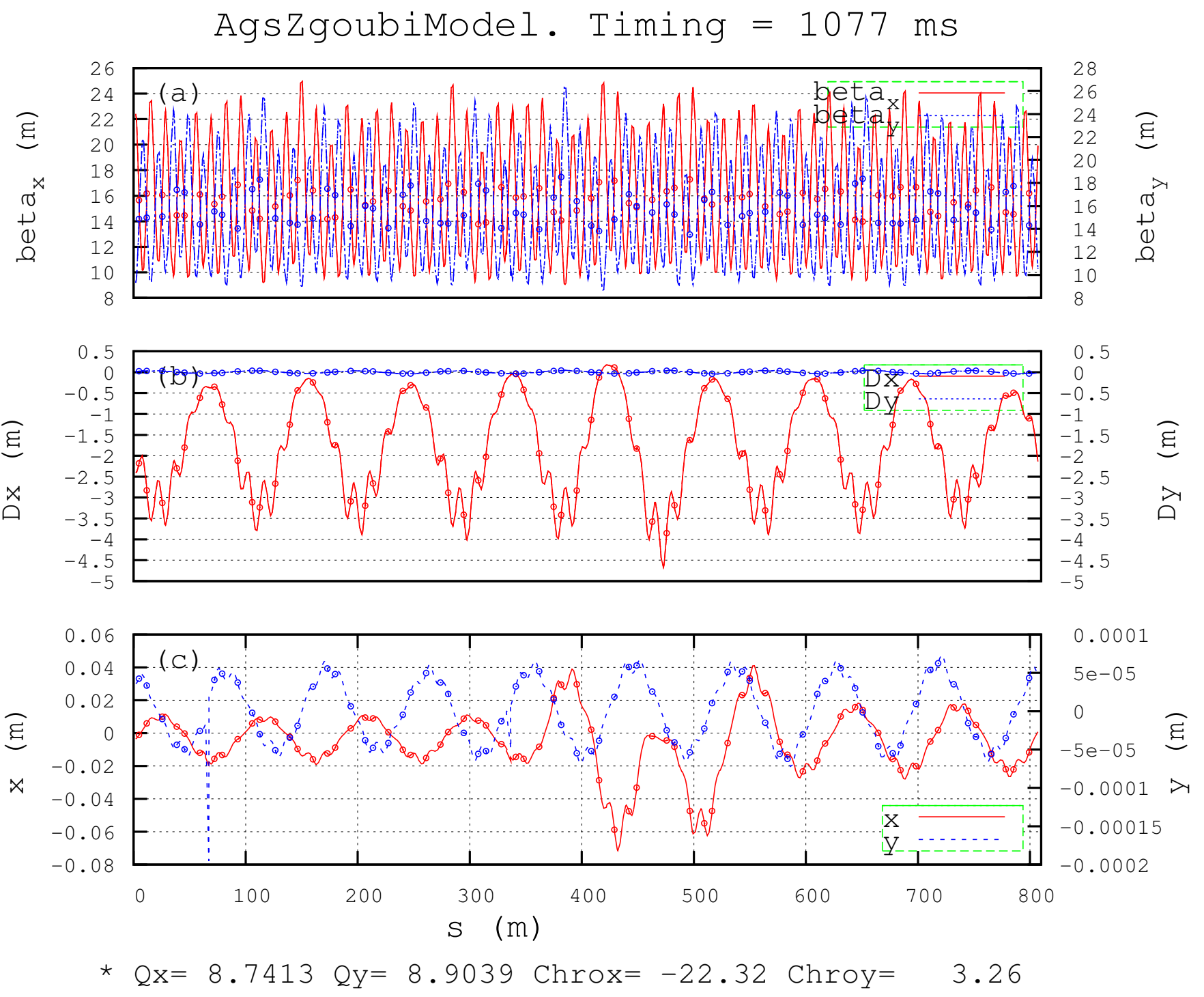

Figure 3: AGS extraction optics (1077 ms after injection, $G \gamma=45.5)$, in presence of the G10 kicker and H10 septum orbit bumps in $350 \lesssim s \lesssim 550 \mathrm{~m}$ - this is for instance the configuration required for determining the periodic spin precession vector $\vec{n}_{0}$ at H10 for transport to RHIC through the AtR, Sec. 4.2 and Fig. 7. Bottom plot: H and V closed orbits are first determined, the vertical orbit (right vertical axis) is very small in the model as an orbit bump is maintained at both snakes, whereas the H10 and G10 bumps are not perfectly closed (they are designed using only two independent power supplies, as found in the snapramp) so causing some horizontal orbit (left vertical axis). Dispersion functions (middle plot) and betatron functions (top plot) are then computed from ray-tracing, with these very $\mathrm{H}$ and V closed orbits taken as Serret-Frenet frame support. Circle markers in these figures show pickup locations. 


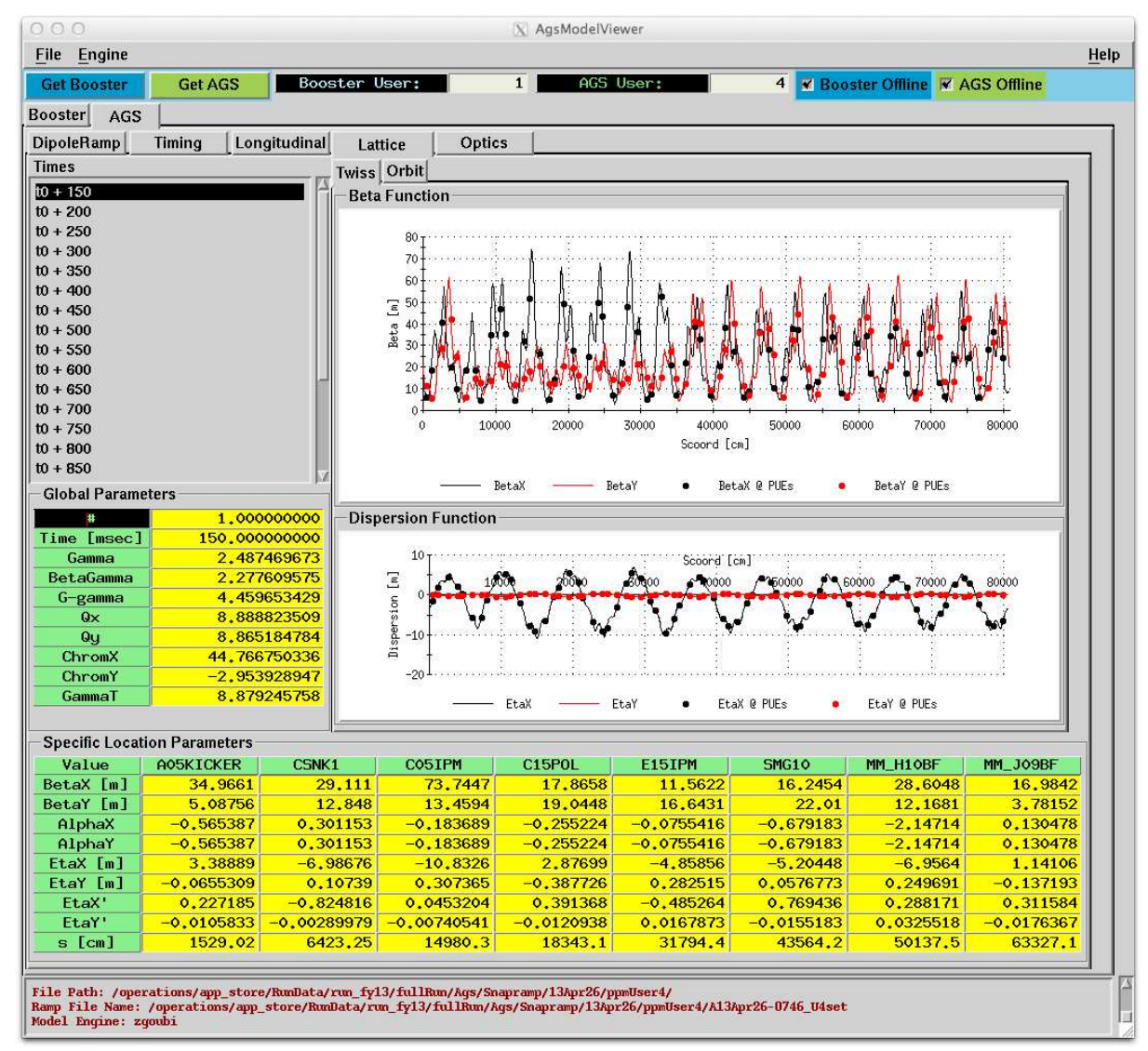

Figure 4: The AGS 'lattice' page of AgsModelViewer, at an early time in the acceleration cycle. AgsModelViewer can use indifferently Zgoubi or MADX engine.

\subsection{AgsModelViewer interface}

The AgsModelViewer [22] is a thin client graphical interface application written in C++ that makes use of a local user interface library developed on top of OSF/Motif® widgets and the commercial XRT ${ }^{\mathrm{TM}}$ (from Quest Software) graphics plotting package. It interfaces to the AGSModelServer, which exists in two instances, one for a MADX model of the AGS and one for the Zgoubi model of the AGS. The servers are generic CDEV servers that collect live or archived data of the machine settings or readbacks and build the instances for the respective model engines. In the case of Zgoubi, the same ZgoubiFromSnaprampCmd infrastructure is used. The model servers are designed to publish model results to any requesting client including command line requests using the cdevCommand interface [27].

The AgsModelViewer is designed to display all the data relevant to the on-line model, see example in Fig. 4, using the earlier snapramp. Once a given set of data is requested, whether from live settings or from an archive of the settings, the viewer will display the magnet currents and other machine settings, and the model output data in terms of Courant-Snyder parameters, generated either from the MADX or Zgoubi server. The viewer can access both. It also contains a simple longitudinal model, with the intent of connecting the longitudinal and transverse models of the accelerators together. This would provide, for example, the momentum compaction factor to the longitudinal model for a more accurate prediction of the synchrotron frequency as a function of time. This feature is part of on-going developments. The viewer is also designed to make comparisons of the model predictions to measurements, so it is capable of reading data logged from instrumentation, such as tune measurements, as a function of time.

\section{THE MODEL OF THE AGS IN Zgoubi}

A model of the AGS in Zgoubi had been first developed based on standard Zgoubi procedures [3, 4], it is still operational, however some specificities of the AGS main magnets and main quadrupoles, such as back-leg windings and other snake compensation quadrupoles, have motivated the development of dedicated elements, 'AGSMM' and 'AGSQUAD', whereas some other Zgoubi procedures as 'OPERA', 'SCALING', have been upgraded. These and all other Zgoubi procedures employed in the AGS model are discussed below. They all are also documented in Zgoubi Users' Guide [28].

\subsection{AGS main magnets - 'AGSMM' and 'MULTIPOL'}

Field strength in the AGS main magnets is drawn from momentum-dependent conversion polynomials (see App. B.2.1). The ad hoc source files have been copied from MADX and installed in Zgoubi. This first lead [3] to simulating AGS main dipoles using an option in MULTIPOL, 
namely, a MULTIPOL is recognized as an AGS combined function magnet by its first label (App. B.2.2). On the other hand, some of the AGS main magnets have back-leg windings, and in addition, it has be seen (Sec. 2.2) that it is desirable to allow perturbing their indices, K1F,D and K2F,D. for fine tune and chromaticity setting purposes. For these various reasons a specific element, AGSMM, was developed, from MULTIPOL, details can be found in App. B.2.2.

\subsection{AGS quadrupoles - 'AGSQUAD'}

Some of the AGS quadrupoles have superimposed windings, driven by separate power supplies. Drawn from MULTIPOL, a specific element was thus developed, AGSQUAD which allows managing quadrupoles with up to three independent windings, and overlapping of quadrupole families, details and example are given in App. B.5.

\subsection{Helical snakes - 'TOSCA'}

The two AGS helical snakes are simulated using 3-D field maps computed using OPERA, and handled using Zgoubi's 'TOSCA' procedure [12]. The orbit at the snake requires two considerations : firstly, it has to evolve during acceleration so to maintain the beam helix across the snake centered on the snake axis (the snakes work at constant field, that ensures minimal variation of spin tilt versus momentum, thus the local orbit bump that ensures helix centering will decrease with increasing energy [12]); secondly, so doing, there remains some horizontal and vertical orbit perturbations, these are cancelled in the model.

Note the following, in that matter : in real life, the sole cold snake undergoes an orbit bump compensation (it is stronger, 2.1 Tesla, compared to $1.5 \mathrm{~T}$ in the warm snake). This orbit bump compensation is zero-ed when the rigidity is high enough that the orbit perturbation by the snake becomes marginal (as a matter of fact, the helix radius decreases with rigidity, it is maximum, $\approx 2.5 \mathrm{~cm}$ in the cold snake, at injection, $G \gamma=4.5$ [12]). This is not the case in the AGS Zgoubi model, so far it has been deemed desirable to work with zero horizontal and vertical orbits over the all AGS ring (apart from the local snake bumps), for that reason the orbit effect of the two snakes is fully compensated, locally, at all energies (see Fig. 31, p. 27).

On the other hand, from the point of view of the model, and essentially in order to allow flexibility in optics and polarization studies, it is desirable that these local orbit bump and residual orbit correction do not encompass the snake-compensation and other tuning quadrupoles located close upstream and downstream of the helix [17], in order to simplify the ramp of the bump during acceleration simulations. However, a local bump encompassing just the snake would involve strong bump dipoles and so jeopardize (i) the path length and (ii) spin motion. To overcome this issue, a trick is used in the model, namely, (i) the snake field map is moved horizontally during acceleration, by an amount which is momentum dependent and ensures the centering of the helix on the snake axis, (ii) the remaining small orbit defect is corrected locally using correctors located right upstream and downstream of the snake, which only require weak field.

Finally, the TOSCA procedure in Zgoubi has been upgraded to linearly superimpose (sum up) several field maps, this capability is used to allow independent current setting (as in real life) in the helical SC dipole and SC solenoid in the cold snake [12].

\subsection{Time varying power supplies - 'SCALING'}

Up to zgoubi-5.1.0 SourceForge download version [29], SCALING only allows varying the field components in a magnet (e.g., dipole to 20-pole in MULTIPOL), all components at once and with all the same scaling factor, for instance during ramps, or for special time-dependent functions. Thus SCALING has been upgraded to allow scaling any data that appears in zgoubi.dat (as is the case for the 'FIT' procedure [28]). This is applied in the AGS Zgoubi model, in a series of commands :

- in AGSMM, in order to independently command (and eventually fit) back-leg winding currents, dB/B, dK1/K1 and dK2/K2 field and index perturbations, injection and extraction orbit bumps (Fig. 3-c), etc.,

- in AGSQUAD, in order to command separate power supplies, in snake compensation quadrupoles for instance, such as the doublewinding quadrupole QP_B03 (App. B.5),

- in the element positioning procedure 'CHANGREF' (a local change of frame) to control the horizontal motion of the two snakes during acceleration.

\subsection{CPU time considerations - integration step size}

Two examples :

- The example of Fig. 2, which features 100 timings about, takes less than a minute to get the un-tuned models, and an additional 4 minutes to get the 100 models tuned to the tune-scan data, on a C-AD computer.

- An acceleration cycle in the AGS from $G \gamma=4.5$ to $G \gamma=45.5$ at a rate of $150 \mathrm{keV}$ per turn, approximately 150000 turns (the actual acceleration rate may slightly differ, depending on operation conditions), takes 2 hours to simulate on a $1.6 \mathrm{GHz} \mathrm{CPU}$, single particle, using $3 \mathrm{~cm}$ stepsize in the AGS main magnets. This is quite convenient in general, for few particle studies on a multipole core office computer, or as well on multiple CPU units. Zgoubi has been installed on NERSC computers [30] for off-line studies, these provide thousands of CPU cores, hence allowing tracking as many particles with similar CPU time - note that some studies are performed launching a few particles per core for more statistics, with still convenient $\approx$ overnight completion. 
In addition, GPU (Graphic Processing Units) possibilities are under investigation, as GPU cards are nowadays commonly available on personal computers and laptops.

\section{Stepsize}

Step size matters in what precedes, as it is the dominant parameter regarding computation time. As a matter of fact, there are 240 main magnets in AGS lattice, thus stepwise integration over their 79 and 94 inches magnetic lengths is the main time-consuming part in code execution; overheads (loading the executable, initializations ...) represent a small fraction of the time budget, negligible when doing singleCPU multi-particle tracking.

It has been shown [11, Section 4.4.1.1] that $1 \mathrm{~cm}$ integration stepsize in the 240 AGS main magnets yields negligible change of Courant invariants (both $\mathrm{H}$ and V) over a 150000 turn AGS acceleration cycle. With $2 \mathrm{~cm}$ stepsize (resp. $3 \mathrm{~cm}$ ) Courant invariant is affected by $<2.5 \%$ (resp. $<10 \%$ - still convenient in many studies).

Zgoubi transports independently of one another all three spin vector components (App. A), the vector has to remain unitary over AGS cycle tracking range, which is the case at better than computer accuracy with integration step size in centimeters scale (see Fig. ?? p. ??, Fig. ?? p. ??).

\section{SAMPLE SIMULATIONS}

Extensive on-line and off-line simulations have been performed using the AGS Zgoubi model, most have been subject to publications in accelerator conferences. Polarization studies specifically, based on 6-D bunch transport through the AGS cycle, can be found in [11], these works are being carried on [31]. Short, sample simulations are reproduced here, aimed mostly at giving a taste of the diversity of the possibilities.

\subsection{Spin motion with tune jumps}

Horizontal intrinsic depolarizing resonances at $G \gamma \pm Q_{x}=$ integer are present in the AGS as the stable spin direction is tilted with respect to the vertical [32, Eq.2.39] as shown in Fig. 5. The 3-periodic structure arises from snake configuration, minimum tilt from vertical in $\mathrm{H} 10$ extraction septum region $(s=500 \mathrm{~m}$, cf. Sec. 4.2) is at $G \gamma=3 n+1.5$. Fig. 6 shows the evolution of the vertical projection of the spin of a single particle at the end of the AGS acceleration cycle $(G \gamma: 40.5 \rightarrow 45.5$ region), with (blue) and without (red) horizontal tune jump. The beneficial effect of tune jumps is apparent.

Similar tracking simulations (single particle as well as thousand particle bunches) along the AGS acceleration cycle, aimed at determining optimal machine conditions for best polarization transmission, are now routinely performed, and serve as a guidance in determining machine settings [34].

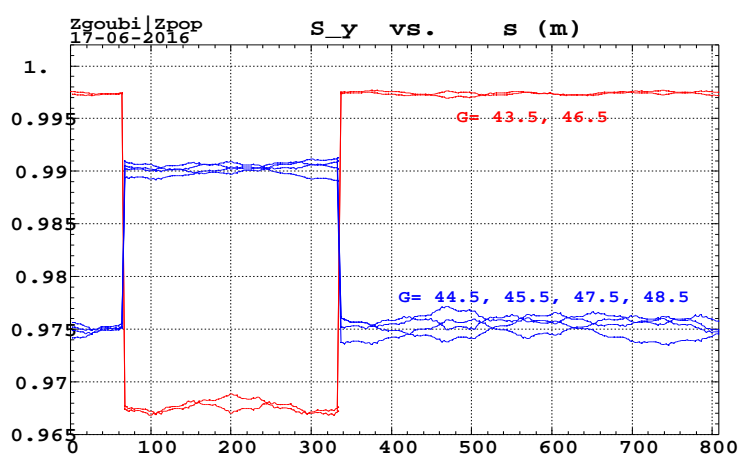

Figure 5: Vertical projection of the spin $\vec{n}_{0}$ vector along the AGS, for various $G \gamma$ values. At extraction $G \gamma=$ 45.5 , the tilt angle from vertical is 8.1 degrees in $65 \lesssim$ $s \lesssim 340 \mathrm{~m}$ and 12.8 degrees over the rest of the ring. The snakes are located at the discontinuities, cold snake to the left and warm snake to the right.

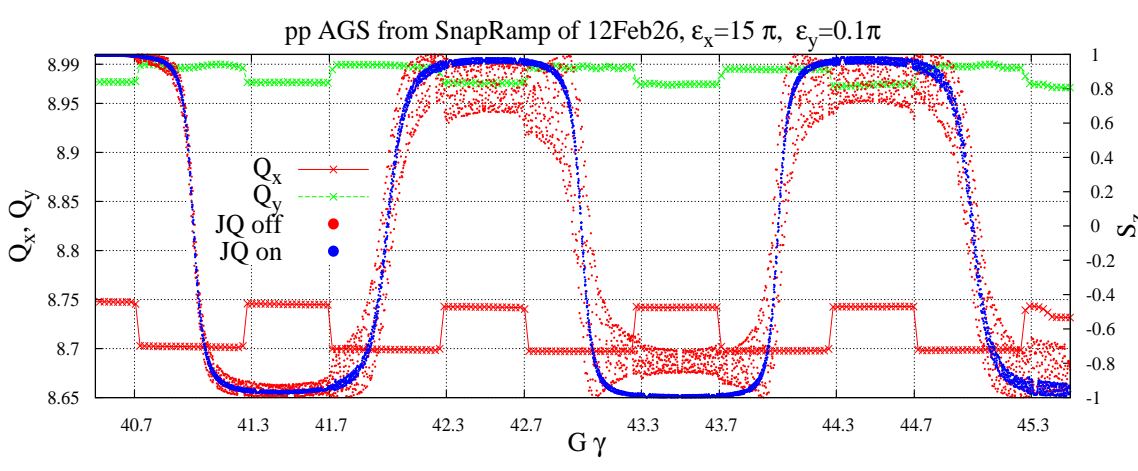

Figure 6: Evolution of $\mathrm{H}$ and $\mathrm{V}$ tunes with $G \gamma$ (left vertical axis) in the presence of horizontal tune jump, and vertical spin component (right axis) with (blue) and without (red) tune jumps. Tune jumps occur at $G \gamma \pm Q_{x}=$ integer, their effectiveness at mitigating depolarization at resonance crossing is apparent. 


\subsection{AGS to RHIC spin matching}

ZgoubiFromSnaprampCmd is used here to generate a model of the AGS, to determine the optimal injection $G \gamma$ into RHIC. The model allows computing the stable spin direction at the AGS extraction septum H10, in the interval $G \gamma: 42 \rightarrow 45.5$. This is the red curve in Fig. 7 (as discussed in Sec. 4.1, the stable $\vec{n}_{0}$ is not vertical in the AGS, Fig. 5). The extraction septum H10 is at $s \approx 500 \mathrm{~m}$, where $\vec{n}_{0}$ is at $\approx 13$ degrees from the vertical at $G \gamma=45.5$ in the conditions of Fig. 7, for instance.

The periodic $\vec{n}_{0}$ is then transported from H10 to RHIC Blue ring injection kicker, and to RHIC Yellow ring kicker, via the AtR (AGS to RHIC transport line, $\approx 600 \mathrm{~m}$ long, with downstream an ' $\mathrm{X}$ ' branch to Blue ring, and a ' $\mathrm{Y}$ ' branch to Yellow ring [14]). This exercise consists in a series of steps, as follows :

(i) get the the periodic orbit in the AGS, including the extraction bumps at G10 and H10 (as in the example in Sec. 4.5),

(ii) get the periodic spin $\vec{n}_{0}$ at H10, after extraction from AGS (G10 kicker on), red curve in Fig. 7,

(iii) transport $\vec{n}_{0}$ along the AtR through RHIC-X and RHIC-Y injection sections down to Blue and Yellow injection kickers, respectively blue and yellow curves in Fig. 7.

Optimal $G \gamma$ value corresponds to $\vec{n}_{0}$ closest to vertical at RHIC Blue and RHIC Yellow kickers (as the stable spin direction in RHIC is vertical). The standard AGS extraction $G \gamma=45.5$ appears close to optimal.

Simulation of phase (iii) requires AtR and RHIC kickers area injection settings, the process of automatizing that and incorporating it in ZgoubiFromSnaprampCmd is underway, it will be based, like for the AGS ring, on the acquisition of AtR snapramp data from a database presently under installation.

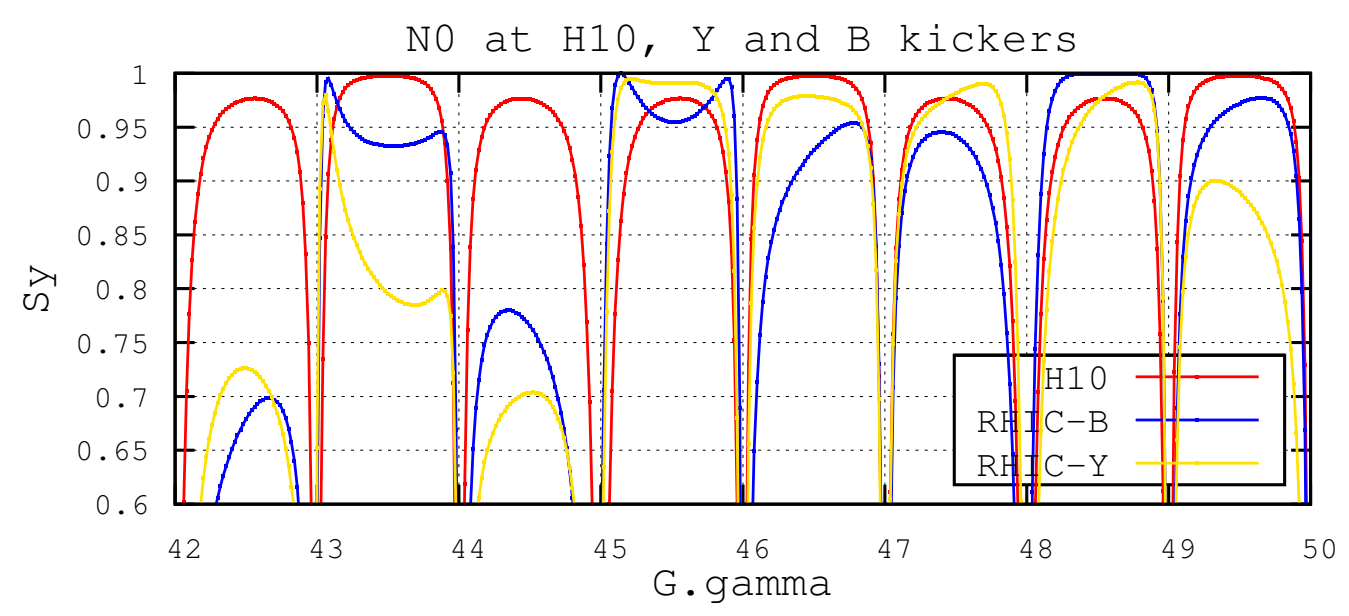

Figure 7: Red curve : vertical component of the periodic $\vec{n}_{0}$ precession vector as observed at septum H10 in AGS, as a function of beam energy (in units of $G \gamma$ ). Blue and Yellow curves : resulting vertical component at the downstream end of RHIC-Blue and RHIC-Yellow injection kickers, after transport of $\vec{n}_{0}$ through the AtR.

The Zgoubi files for this exercise have been archived at

$$
\text { /rap/lattice_tools/zgoubi/AgsZgoubiModel/AtR/n0 }
$$

Studies regarding the effects of orbit defects or large beam emittance on the transmission of ${ }^{3} \mathrm{He}^{2+}$ beam polarization along the AtR, in view of future RHIC and eRHIC project programs, have been performed recently, using these tools [15].

\subsection{Orbit with survey data}

AGS main magnet survey data have been included in the Zgoubi model for the sake of comparisons with MADX investigations [35]. Defects accounted for include $\delta x$ and $\delta y$ positioning errors, pitch, yaw and roll rotations. Sample outcomes and comparisons are displayed in Fig. 8. More on the outcomes from these survey data can be found in [36].

Including survey data opens the way to orbit simulations. This is in the plans for future developments of the AGS model in Zgoubi.

The Zgoubi files for this exercise have been archived at

$$
\text { /rap/lattice_tools/zgoubi/AgsZgoubiModel/AgsZgoubiModel_examples/agsWithSurveyData/ }
$$

\subsection{Non-linear coupling with snakes}

Fig. 9 shows a simulation in which the tunes, starting close to the diagonal, are then moved onto the $Q_{x}-2 Q_{y}$ resonance line in a few $10^{4}$ turns, at low, constant, energy. Coupled motion causes modulation of the betatron amplitude as the particle approaches and stands on 

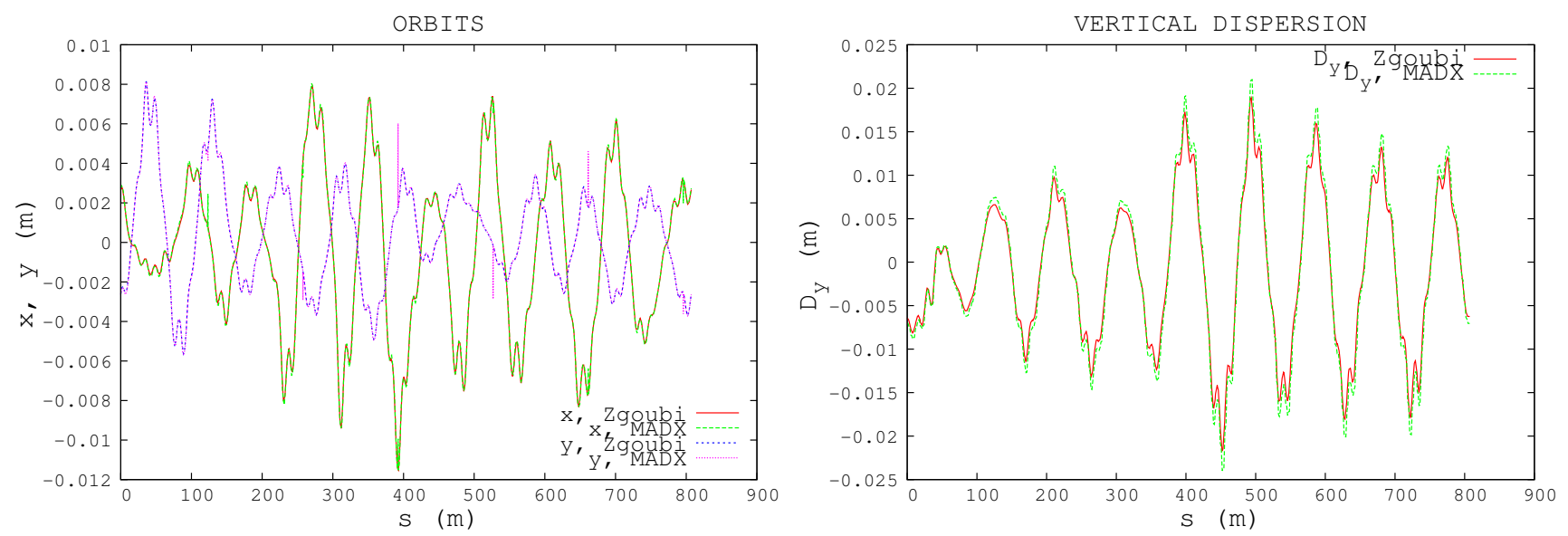

Figure 8: Left : closed orbit in the AGS model resulting from introduction of alignment survey data. Right : vertical dispersion. Zgoubi and MADX models are superimposed to show their agreement.

that non-linear resonance line. That resonance line is excited by normal sextupole field component from the 2.1 Tesla cold snake, the effect disappears if the cold snake is turned off (main magnet sextupole fields have negligible effect, whereas it is much weaker with the 1.53 Tesla warm snake). The field in the helical dipole, responsible for that, writes [37, Eq. 5].

$$
\begin{gathered}
b_{x}=-\sin \phi+\frac{1}{8} k^{2}\left(3 x^{2}+y^{2}\right) \sin \phi+\frac{1}{4} k^{2} x y \cos \phi \\
b_{y}=\cos \phi-\frac{1}{8} k^{2}\left(x^{2}+3 y^{2}\right) \cos \phi+\frac{1}{4} k^{2} x y \sin \phi \\
b_{z}=k y \sin \phi+k x \cos \phi
\end{gathered}
$$

with $r=\sqrt{x^{2}+y^{2}}$ in the centimeter (low energy) to millimeter range (high energy).
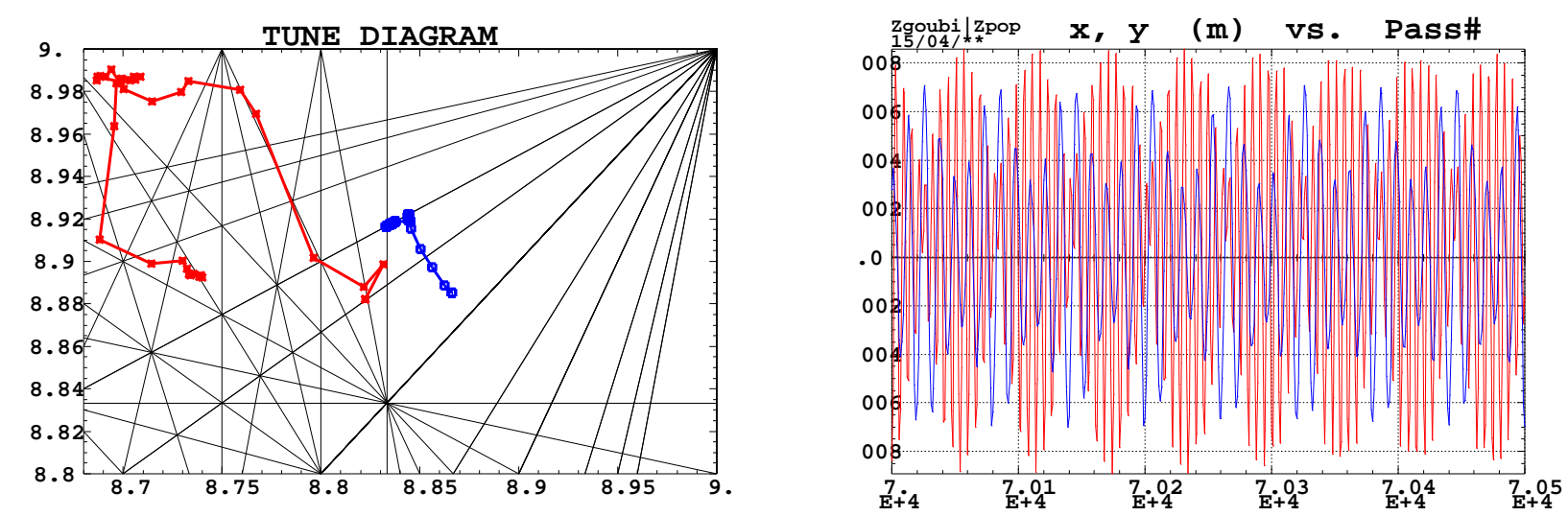

Figure 9: Left : beam path in the tune diagram over AGS acceleration cycle (red line), and in the present case of coupling exploration (blue track ending on $Q_{x}-2 Q_{y}$ ). Right : modulation of the betatron motion is observed (x and $\mathrm{y}$ in phase opposition) once the particle straddles the resonance line, turns number $7 \times 10^{4} \rightarrow 7.05 \times 10^{4}$.

The dipole symmetry non-linearities in the snakes (sextupole, decapole, etc.) are stronger at larger radius, hence at lower rigidity as the radius of the helix in the snake decreases (linearly) with momentum [12, Figs. 2, 8, 13]. Note that, due to the helical geometry the skew fields are also present (Eq. 1).

The exercise of moving the beam on non-linear coupling lines has been performed in the AGS during RHIC runs 13-15, to investigate qualitatively possible effects at low energy, in the presence of the snakes, as indicated by the simulations. The experimental results span several beam energy values in the low energy range and confirm these simulations, they can be found in the AGS eLogs and will be subject to future AGS Tech.-Note. Zgoubi files for these simulations have been archived at

$$
\text { /rap/lattice_tools/zgoubi/AgsZgoubiModel/AgsZgoubiModel_examples/AGSOptics/coupling/qx.84qy.92(qx-2qy) }
$$

\subsection{AGS extraction optics}

An AGS technical note on the AGS Extraction is available [38]. The study uses the Raytrace code and the measured field maps of the AGS magnets. This technical note contains detailed information on the extraction bumps and the G10 kicker, and on the main magnet fringe fields included in the extraction calculations. An AGS Zgoubi model which reproduces these extraction conditions (horizontal G09 and H11 bumps 
as in Fig. 3-c) has been archived at

/rap/lattice_tools/zgoubi/AgsZgoubiModel/AgsZgoubiModel_examples/AGSOptics/ExtractionBumps_2016 - 06 - 20

comments can be found in the README file therein. It uses the AGS snapramp

/operations/app_store/RunData/run_fy15/fullRun/Ags/Snapramp/15Mar08/ppmUser4/15Mar08 - 0854_fgm_U4cur/

There are essentially two methods to obtain the bump from ZgoubiFromSnaprampCmd, as follows.

First method :

(i) set timing to $1077 \mathrm{~ms}$ in ZgoubiFromSnaprampCmd.in (a template is given in App. D.1),

(ii) run ZgoubiFromSnaprampCmd.

This generates model_1077.zgoubi (renamed [...]/ExtractionBumps_2016-06-20/model_1077.zgoubi_G09-H11_byHand), and this folder contains a model of the AGS at that timing, namely, zgoubi.dat.

(iii) By default, the backleg windings for G09 and H11 extraction bumps are set to very low currents (this is the case in the template zgoubi.dat file given in App. D.3, it can be seen there that currents are set to, respectively, $-.0762 \times 10^{-99}$ and $-.06093 \times 10^{-99}$ Amp). Hence the next step,

(iv) edit zgoubi.dat, and set the G09 current to -.0762 Amp, the H11 current to -.06093 Amp.

(v) This much Amps in G09 and H11 main magnet backleg windings will trigger the required, non-zero, extraction orbit. Actual orbit coordinates can be found either using the multi-turn ('REBELOTE') procedure in Zgoubi, or the 'FIT' procedure [28].

Second method :

What precedes can actually be managed by ZgoubiFromSnaprampCmd (so skipping steps (iv), (v)), by

a/ setting to 1 the 'bump switch' under '[AGS MODEL DATA]', in ZgoubiFromSnaprampCmd.in (App. D.1), and

$\mathrm{b} /$ setting to 1 the 'closed orbit search' switch in the following line, this is the example saved

in [...]/ExtractionBumps_2016-06-20/model_1077.zgoubi.

This yields a MADX style 'twiss' file, zmodel_1077.zgoubi.twiss (saved by ZgoubiFromSnaprampCmd in model_1077.zgoubi/twiss folder). Plotting its content yields the optical functions and orbits in Fig. 10.
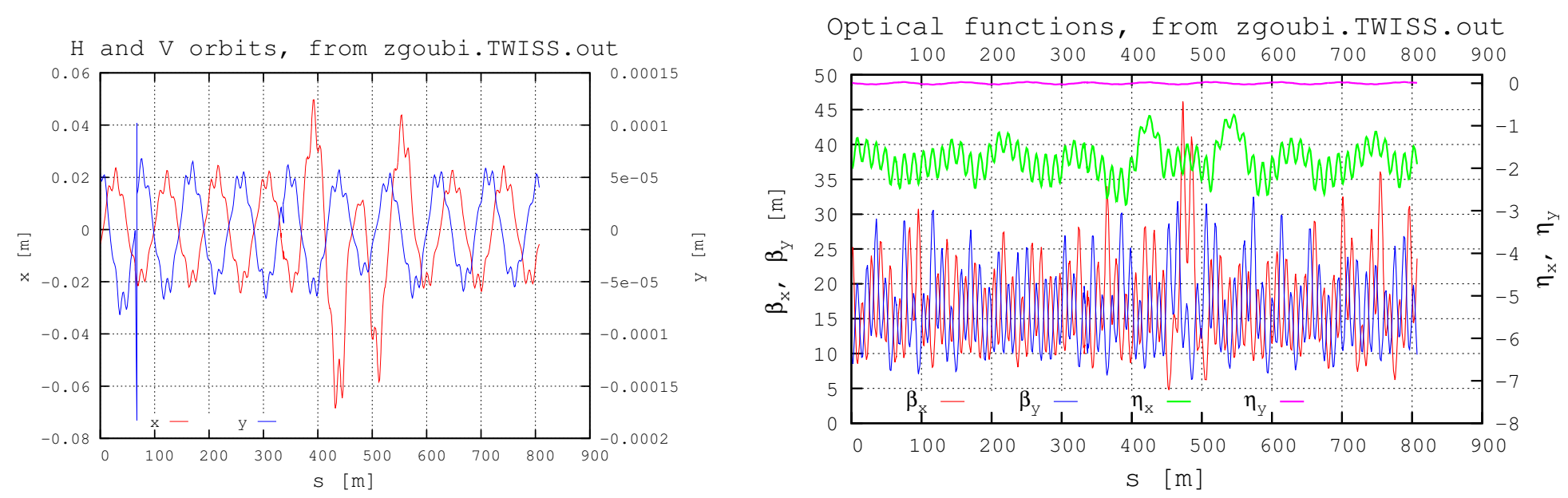

Figure 10: Orbits (left) and optical functions (right) resulting from -.0762 Amp G09 bump and -.06093 Amp H11 bump excitation, in an AGS Zgoubi model straight out of ZgoubiFromSnaprampCmd. 


\section{WHERE ARE THE FILES ?}

\subsection{Template input data file to ZgoubiFromSnaprampCmd}

ZgoubiFromSnaprampCmd requires either an input data file, ZgoubiFromSnaprampCmd.in (App. D.1), or arguments (in a similar manner to MadxFromSnaprampCmd (App. D.2). A template of the former is stored at

$$
\text { /rap/lattice_tools/zgoubi/AgsZgoubiModel/templateZgoubiFromSnaprampCmd.in_library/ }
$$

If ZgoubiFromSnaprampCmd.in is not found in the folder where ZgoubiFromSnaprampCmd is launched, then this template is taken by default. App. D.1 details the content of ZgoubiFromSnaprampCmd.in.

\subsection{Template Zgoubi AGS sequence file}

In order to build a model of the AGS, ZgoubiFromSnaprampCmd requires a template zgoubi.dat file that contain the AGS sequence, 'templateZgoubi4ZgoubiFromSnaprampCmd.dat'.

Two templates are maintained at

$$
\text { /rap/lattice_tools/zgoubi/AgsZgoubiModel/templateZgoubi4ZgoubiFromSnaprampCmd_library/ }
$$

for respectively polarized protons and ions, namely

$$
\begin{aligned}
& \text { ppmU ser4_pp/templateZgoubi4ZgoubiFromSnaprampCmd } \\
& \text { ppmUser1/templateZgoubi4ZgoubiFromSnaprampCmd.dat }
\end{aligned}
$$

If templateZgoubi4ZgoubiFromSnaprampCmd.dat is not found in the folder where ZgoubiFromSnaprampCmd is launched, then one of these two is taken by default, that consistent with particle specifications in ZgoubiFromSnaprampCmd.in (App. D.1).

\subsection{Helical snake field maps}

New OPERA field maps of the cold snake have been computed, with various helix and/or solenoid current settings [12], they have been archived in dedicated folders in

$$
\text { /rap/lattice_tools/zgoubi/AgsZgoubiModel/snakeFieldMaps/coldSnake/RGFiles_Mar2013 }
$$

The other, original, OPERA field maps of the cold snake have been archived in additional dedicated folders in

$$
\text { /rap/lattice_tools/zgoubi/AgsZgoubiModel/snakeFieldMaps/coldSnake/ }
$$

New warm snake OPERA maps have also been computed, with finer mesh in order to overcome particle dynamics issues experienced with original maps [11]. All presently available maps have been archived in

$$
\text { /rap/lattice_tools/zgoubi/AgsZgoubiModel/snakeFieldMaps/warmSnake/ }
$$

README files in these various folders give a guidance as to the respective properties and usage of the various maps.

\subsection{AGS main magnets field maps}

Measured field maps of the different types of AGS main magnets are available, their original versions have been archived at

$$
\text { /rap/lattice_tools/zgoubi/AgsZgoubiModel/mainMagnetFieldMaps/originalFilesFromNick }
$$

A smoothed field version of these maps has been worked out for Zgoubi for mainly two reasons which are, (i) have a uniform mesh size, (ii) get a smoother field (reduced fluctuations by smoothing the measurements), this is discussed in App. B.3.1. These Zgoubi compatible versions are stored at

$$
\text { /rap/lattice_tools/zgoubi/AgsZgoubiModel/mainMagnetFieldMaps/6MapsOkForZgoubi }
$$

\subsection{Zgoubi source and executable}

ZgoubiFromSnaprampCmd runs the models it fabricates using

$$
\text { /rap/lattice_tools/zgoubi/zgoubi }
$$

This executable is maintained specifically for the booster [25] and AGS models. Physically this executable is located in the folder/rap/lattice_toc the source files are at

$$
\text { /home/owl/fmeot/zgoubi/SVN/AgsZgoubiModel_source_copies/ }
$$

Older versions of the executable are archived in /rap/lattice_tools/bin/ as well, whereas /rap/lattice_tools/zgoubi/zgoubi is normally a pointer to the most recent one.

However Zgoubi can also be downloaded from SourceForge open source projects site [29], the version available there is maintained compatible with the AGS model. 


\subsection{ZgoubiFromSnaprampCmd and ancillary source files}

ZgoubiFromSnaprampCmd source files are in

$$
\text { /rap/lattice_tools/zgoubi/agszgoubimodel/sources }
$$

Found in that folder also are various ancillary programs that the command uses, e.g., gnuplot files (in gnuplotTemplates or in various sub-folders).

Examples of ZgoubiFromSnaprampCmd run outcomes can be found in

$$
\text { /rap/lattice_tools/zgoubi/AgsZgoubiModel/AgsZZgoubiModel_examples/ }\left\{\begin{array}{l}
\text { Example_2012-08 - 01 } \\
\text { Example_2015-05-17 }
\end{array}\right.
$$

\section{CONCLUSION}

The AGS Zgoubi model is based on stepwise ray-tracing methods and allows detailed simulations and studies regarding optical properties of, and polarized bunch dynamics in RHIC injector, the AGS ring. It also allows investigating individual magnets, e.g., AGS main magnets, helical snakes, development of new instrumentation, etc.

The AGS Zgoubi model adds to the RHIC model in Zgoubi which has proven efficient in simulating snake resonance crossing up to high precession rates at $255 \mathrm{GeV}$ RHIC top energy [20] ( $G \gamma=487$ spin precessions over one RHIC turn, of the order of 2+ precessions per bend), and for future RHIC and eRHIC project programs [39, 40], and it adds to the Booster model under development [25], used in the recent past for the simulation of polarized helion transport in view of He-p collisions in RHIC [41]. This confirms the Zgoubi method as an efficient tool for the modeling polarized beam transport in RHIC accelerator complex, and for studies in relation with future projects and spin physics programs at BNL. 


\section{APPENDIX}

\section{A Zgoubi method, in short}

The integration method in Zgoubi is based on stepwise integration of the Lorentz equation using a Taylor series technique : position and velocity vectors of a particle at location $M_{1}(s+\Delta s)$ after a step $\Delta s$ are computed from Taylor expansion of position and velocity at earlier location $M_{0}(s)$, Fig. 11,

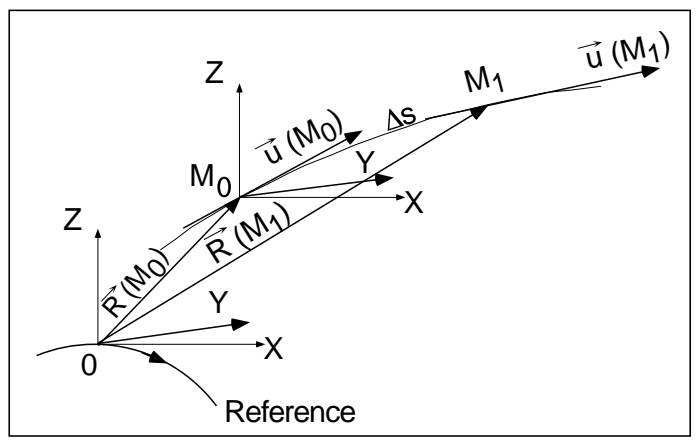

Figure 11: Position and velocity of a particle in Zgoubi reference frame.

$$
\vec{R}\left(M_{1}\right)=\vec{R}\left(M_{0}\right)+\sum_{n=1}^{N} \frac{d^{n} \vec{u}}{d s^{n}} \frac{\Delta s}{n !}, \quad \vec{u}\left(M_{1}\right)=\vec{u}\left(M_{0}\right)+\sum_{n=1}^{N-1} \frac{d^{n} \vec{u}}{d s^{n}} \frac{\Delta s}{n !}
$$

The Taylor coefficients are the derivatives of the velocity and drawn from the Lorentz equation, namely,

$$
\begin{aligned}
& (B \rho)^{\prime} \vec{u}+B \rho \vec{u}^{\prime}=\frac{\vec{e}}{v}+\vec{u} \times \vec{b}, \quad \text { this yields } \vec{u}^{\prime} \\
& (B \rho)^{\prime} \vec{u}+2(B \rho)^{\prime} \vec{u}^{\prime}+B \rho \vec{u}^{\prime}=\left(\frac{1}{v}\right)^{\prime} \vec{e}+\left(\frac{1}{v}\right) \vec{e}^{\prime}+(\vec{u} \times \vec{b})^{\prime}, \quad \text { this yields } \vec{u}^{\prime} \\
& \text { and so forth for } \quad u^{\prime \prime}, u^{\prime \prime \prime} \ldots
\end{aligned}
$$

wherein

$$
(*)^{\prime}=\frac{d(*)}{d s}, \quad \vec{u}=\frac{\vec{v}}{v}, \quad d s=v d t, \quad \vec{u}^{\prime}=\frac{d \vec{u}}{d s}, \quad m \vec{v}=m v \vec{u}=q B \rho \vec{u}, \quad \vec{b}=\vec{B} / B \rho, \quad \vec{e}=\vec{E} / B \rho
$$

with $B \rho$ is the rigidity of the particle, $m$ its mass, charge $q$, velocity $\vec{v}$, magnetic and electric fields $\vec{B}$, $\vec{E}$. The integration thus requires the knowledge - and the modeling - of the magnetic/electric field and its derivatives. Details of the method can be found in [1].

Spin motion is treated in a similar manner, using the previous field ingredients, namely

$$
\vec{S}\left(M_{1}\right)=\vec{S}\left(M_{0}\right)+\sum_{n=1}^{N} \frac{d^{n} \vec{S}}{d s^{n}} \frac{\Delta s}{n !}
$$

with $\vec{S}^{\prime}$ and the recurrent differentiation $\left(\vec{S}^{\prime}, \vec{S}^{\prime \prime}\right.$, etc.) drawn from [42]

$$
\frac{d \vec{S}}{d t}=\frac{q}{m} \vec{S} \times \vec{\omega} \text { or, } \vec{S}^{\prime}=\vec{S} \times \vec{\Omega} \text {, with } B \rho \vec{\Omega}=\omega=(1+\gamma G) \vec{b}+G(1-\gamma) \vec{b}_{\|}+\gamma\left(G+\frac{1}{1+\gamma}\right) \frac{\vec{e} \times \vec{v}}{c^{2}}
$$

\section{B Model of the AGS in Zgoubi}

\section{B.1 The OCO}

A reference line, 'OCO' (Optimum Closed Orbit), has been defined in the AGS, Figs. 12, 13 [43, 44]. The OCO coincides with the closed orbit in the straight sections between the 240 main dipoles. In the dipoles themselves, the OCO coincides with the chord of the arc of trajectory, it is localized by its distance to the socket line (the survey line). OCO coincides with the optical axis of elements placed in the drifts, as tuning quads, sextupoles, control instrumentation, etc.

The model of the AGS main magnet in Zgoubi sticks to these principles, as shown in the next section : the closed orbit in Zgoubi coincides with the OCO line, all lenses introduced further in the lattice are by default (i.e., in the absence of explicit request for a different alignment) centered on that line. 


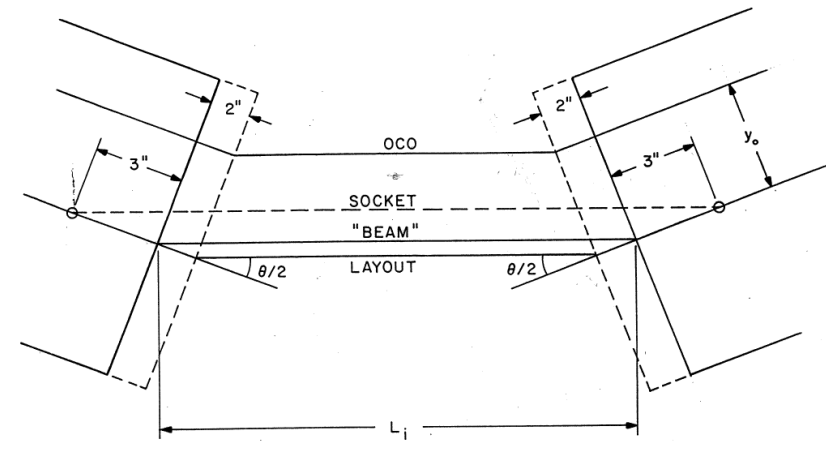

Figure 12: OCO line in the AGS, the reference optical axis [43, Fig. A1]. The 2" distance at magnet ends is between the iron and the effective field boundary (EFB). $y_{0}$ denotes the distance between the cord of the orbit arc in the magnet, and the survey socket line.
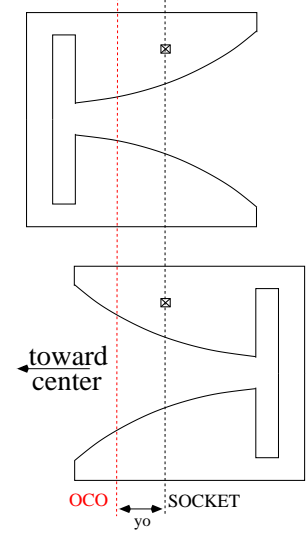

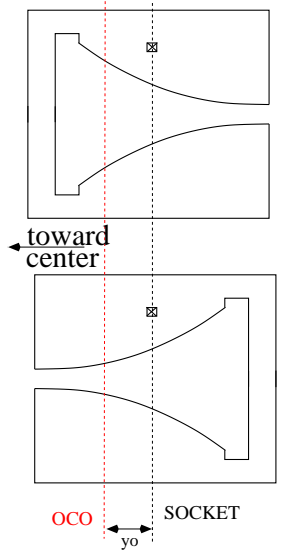

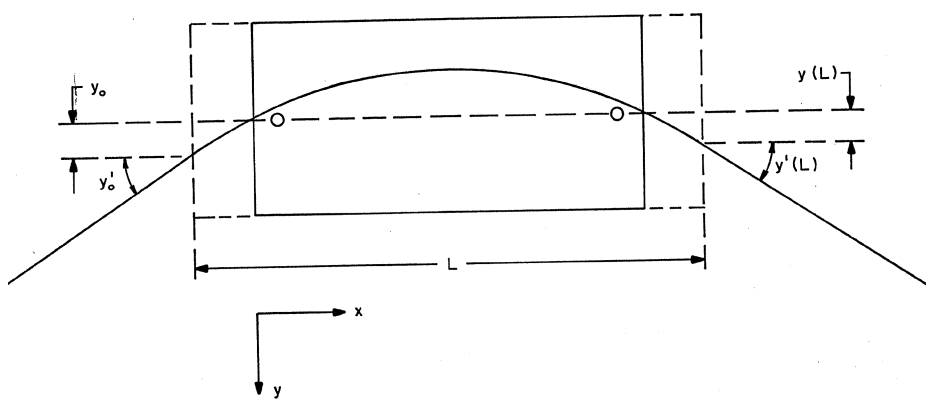

Figure 13: This figure [43, Fig. 1] shows the symmetric placement of the OCO, in position $y_{o} \equiv y(L)$, and in angle $y_{0}^{\prime} \equiv y^{\prime}(L)$ with respect to the socket line. This yields arc length and deviation as given in Tab. 2. The quantity $y_{0}$ in this and Fig. 12 identifies with $\Delta x$ in Tab. 2.

Figure 14: A and $\mathrm{C}$ type of magnet cross sections. A and $\mathrm{C}$ are 90 inches long (iron length). B type is a shorter versions of $\mathrm{A}, 75$ inches long (iron length). The figure shows that $\mathrm{AD}, \mathrm{BD}, \mathrm{CD}$ are defocusing, $\mathrm{AF}$, $\mathrm{BF}, \mathrm{CF}$ are focusing.

\section{B.2 An analytical model of the AGS main magnet field}

There are six different types of straight-axis combined function dipoles in the AGS ring, Fig. 14, for a total of 240, 20 per superperiod, 12 superperiods. The momentum-dependent quadrupole and sextupole strengths in these six magnet types are represented by as many polynomials, $K_{1}(p), K_{2}(p)$. They have been taken from the MAD model of the AGS [24, 45], and installed in Zgoubi source code as part of the definition of the AGS combined function dipole. $K_{1}(p), K_{2}(p)$ account for the variation of the field in the gap during the ramp. On the other hand a classical multipole scalar potential model is used for AGSMM, AGSQUAD and MULTIPOL in Zgoubi (see next Secs.), namely [28, Sec. 1.3.7]

$$
\begin{array}{r}
\text { - dipole: } V_{1}(s, x, y)=D(s) y-\frac{D^{\prime}(s)}{8}\left(x^{2}+y^{2}\right) y+\frac{D^{\prime \prime}(s)}{192}\left(x^{2}+y^{2}\right)^{2} y-\ldots, \quad D(0)=B_{0} \\
\text { - quadrupole : } V_{2}(s, x, y)=G(s) x y-\frac{G^{\prime}(s)}{12}\left(x^{2}+y^{2}\right) x y+\frac{G^{\prime \prime}(s)}{384}\left(x^{2}+y^{2}\right)^{2} x y-\ldots, \quad G(0)=B_{1} / R \\
\text { - sextupole: } V_{3}(s, x, y)=\frac{H(s)}{3}\left(3 x^{2}-y^{2}\right) y-\frac{H^{\prime}(s)}{48}\left(3 x^{4}+2 x^{2} y^{2}-y^{4}\right) y+\ldots, \quad H(0)=B_{2} / R^{2}
\end{array}
$$

$D, G, H(s)$ coefficient in $V_{1}-V_{3}$ has the role of a longitudinal form factor and allows simulating soft field fall-offs, using for instance an Enge model [28, Sec. 1.3.7],

$$
D, G, H(s)=\frac{1}{1+\exp (P(d))}, \quad P(d)=C_{0}+C_{1} \frac{d}{\lambda}+C_{2}\left(\frac{d}{\lambda}\right)^{2}+\ldots+C_{5}\left(\frac{d}{\lambda}\right)^{5}
$$

with $d$ the distance to the magnet effective field boundary (EFB), and $C_{0}-C_{5}$ coefficients that determine the length and shape of the fall-off (Fig. 28, p. 25). In a hard-edge end-field model, $D, G, H(s)$ is a Heaviside step, thus the expressions above simplify to

$$
V_{1}=D y, \quad V_{2}=G x y, \quad V_{3}=\frac{H}{3}\left(3 x^{2}-y^{2}\right) y
$$

with D, G, H constants. 


\section{B.2.1 Centered and off-centered multipole models}

Modeling the AGS combined function dipole normally uses AGSMM keyword, however it can also use the standard Zgoubi routine MULTIPOL [7] from which the former was derived (App. B.2.2). Both keywords are documented in Zgoubi Users' Guide [28].

The transverse field components in this multipole model are derived from the solution of Laplace equation, including possible longitudinal dependence (D(s), G(s), H(s) quantities in Eq. 7).

For all six types of magnets, Fig. 14, the value of the dipole component $B_{0}(p)$ ensures momentum-independent orbit arc geometry in the magnet, it derives from the geometry of the OCO [44, App. IV], as follows.

The straight magnetic lengths in A- (or C- )and B-type magnets are respectively 94 inches $(2.3876 \mathrm{~m})$ and 79 inches $(2.0066 \mathrm{~m})$. The corresponding arc lengths are 94.0030604 inches $(2.387678 \mathrm{~m})$ and 79.0018181 inches $(2.006646 \mathrm{~m})$, respectively [44, App. IV]. The bending angle values are given in Tab. 2, col. 4.

The overall arc length from Zgoubi ray-tracing, col. 3 in Tab. 2, results in a bending radius of ${ }^{1}$

$$
[\underbrace{72 \times 2.387678}_{72 \text { long } F}+\underbrace{72 \times 2.387677}_{72 \text { long } D}+\underbrace{48 \times 2.006646}_{48 \text { short } F}+\underbrace{48 \times 2.006595}_{48 \text { short } D}] / 2 \pi=85.380440
$$

Arc lengths as obtained from ray-tracing in Zgoubi model are given in Tab. 2, col. 3, in very good accord with Refs [43, 44, 48].

The quadrupole strengths $\mathrm{K} 1 \mathrm{AD}$, - $\mathrm{AF}$, -BD, -BF, -CD, -CF are drawn from momentum-dependent polynomials of the form [45]

$$
K 1(p)=q_{-2} / p^{2}+q_{-1} / p+q_{0}+q_{1} \times p+q_{2} \times p^{2}+q_{3} \times p^{3}+q_{4} \times p^{4}+q_{5} \times p^{5}+q_{6} \times p^{6}
$$

Similarly, for the residual K2 sextupole strength series K2AD, -AF, -BD, -BF, -CD, -CF,

$$
K 2(p)=s_{-3} / p^{3}+s_{-2} / p^{2}+s_{-2} / p+s_{0}+s_{1} \times p+s_{2} \times p^{2}+s_{3} \times p^{3}+s_{4} \times p^{4}+s_{5} \times p^{5}+s_{6} \times p^{6}
$$

We dispose of two series of coefficients for these K1(p) and K2(p) polynomials. A first one is that currently in use in the MAD model of the AGS and has its origin as described in [45]. The second series has been derived, as part of the AGS Zgoubi model developments, from the measured field maps of the main dipole [49] (App. B.3.2), both series provide very similar strengths (see Fig. 27).

Note that translators from MAD8 to Zgoubi, and from MADX to Zgoubi, written in Fortran, are available [50]. These polynomials have been installed in both translators at an early stage of these developments, and allowed straightforward comparisons between the two codes. However such comparisons can be done using AgsModelViewer which allows for either one of the two engines, Zgoubi or MADX.

Table 2: AGS main magnet parameters. Cols. 2, 3, 4 : arc lengths from [44] and from Zgoubi ray-tracing, bend angle, for the six different magnet types. Col. 5 : magnet alignment in Zgoubi, to satisfy OCO and first order mapping requisites ; values from [44,

\begin{tabular}{|c|c|c|c|c|c|c|c|c|}
\hline & \multirow{2}{*}{$\begin{array}{l}\text { Arc length } \\
\text { from } \\
\text { Ref. [44] } \\
\text { (m) }\end{array}$} & \multirow{2}{*}{$\begin{array}{l}\text { Arc length } \\
\text { from } \\
\text { Zgoubi } \\
\text { ray-tracing } \\
\text { (m) }\end{array}$} & \multirow[b]{2}{*}{$\begin{array}{c}\theta \\
(\mathrm{mrad})\end{array}$} & \multicolumn{2}{|c|}{$\begin{array}{c}\Delta x \\
\text { 'centered-multipole' model }\end{array}$} & \multirow{2}{*}{$\begin{array}{c}\Delta x_{\mathrm{off}} \\
\text { 'shifted- } \\
\text { multipole' } \\
\text { model } \\
(\mathrm{cm})\end{array}$} & \multicolumn{2}{|c|}{$\begin{array}{c}\text { Sample values } \\
\text { taken at } G \gamma=4.5\end{array}$} \\
\hline & & & & $\begin{array}{l}\text { From ray- } \\
\text { tracing) }\end{array}$ & $\begin{array}{c}\text { From } \\
{[44, \text { Tab. 1] }} \\
(\mathrm{cm})\end{array}$ & & $\begin{array}{c}K_{1} \\
\left(10^{-2} \mathrm{~m}^{-2}\right)\end{array}$ & $\begin{array}{c}K_{2} \\
\left(10^{-3} \mathrm{~m}^{-3}\right)\end{array}$ \\
\hline $\mathrm{BF}$ & 2.006646 & 2.006647 & 23.5023 & -0.3942 & 0.3929 & 23.17 & 4.848 & 5.056 \\
\hline $\mathrm{CD}$ & 2.387678 & 2.387677 & 27.96503 & -0.5539 & 0.5565 & -24.07 & -4.866 & 4.378 \\
\hline $\mathrm{AF}$ & 2.387678 & 2.387679 & 27.96503 & -0.5589 & 0.5565 & 23.00 & 4.868 & 4.362 \\
\hline $\mathrm{BD}$ & 2.006646 & 2.006595 & 23.5023 & -0.3917 & 0.3929 & -23.93 & -4.844 & 5.002 \\
\hline $\mathrm{CF}$ & 2.387678 & 2.387679 & 27.96503 & -0.5589 & 0.5565 & 23.00 & 4.873 & 4.423 \\
\hline $\mathrm{AD}$ & 2.387678 & 2.387677 & 27.96503 & -0.5539 & 0.5565 & -24.07 & -4.863 & 4.305 \\
\hline
\end{tabular}
$y_{0}$ value in Fig. 3 and App.II] in col. 6 are for comparison. Col. 7 : orbit off-centering in the 'shifted-quadrupole' model drawn from OPERA field maps. Cols. 8, 9 : typical quadrupole and sextupole strengths (here, values for $B \rho=7.20517$ T.m).

Finally, AGSMM (and MULTIPOL as well) model in Zgoubi allows the simulation of perturbative effects of all sorts as length or field defects, misalignements, high order multipoles.

Centered multipole - This model assumes the superimposition of independent dipole, quadrupole and sextupole field components (Eq. 7) all with a common multipole axis.

The dipole field component for the six different types of dipoles is determined from the geometry of the OCO as described above, the field strengths K1, K2 are computed using Eqs. 10, 11.

In addition, all six magnet types have to be slightly shifted, by a small amount $\Delta x$, column 5 in Tab. 2, determined by ray-tracing, in order to cause their transfer matrices as of Zgoubi to identify with MAD ones. Satisfyingly, that amount happens to be practically identical with the $y_{0}$ shift in Bleser's Fig. 3, value given in Appendix II, Ref. [44] and reproduced for comparison in the present Tab. 2, col.6.

This model yields very good agreement with MADX, at all timings, see for instance tune scan in Fig. 20 and details at $G \gamma=45.5$ in Tab. 3, cols.2, 3 .

\footnotetext{
${ }^{1}$ This result is reasonable, considering earlier published data : a bending radius of 3361.4503 inches, $85.380838 \mathrm{~m}$, is obtained with the 94.0030604 in. and 79.0018181 in. arc lengths of [44, App. IV] (a $0.4 \mathrm{~mm}$ difference with Eq. 9). A radius of $85.380840 \mathrm{~m}$ is quoted, later, in [46, 47, page 2] using lengths taken from [44], stating in addition 'This value should be consistent with other calculations to about 0.5 millimeters'.
} 
Off-centered multipole - This model assumes the dipole component $B_{0}$ to be due to the OCO being positioned at an ad hoc distance $\Delta x_{\mathrm{Off}}$ from the axis in a quadrupolar field, as sketched in Fig. 15, namely,

$$
B_{0}=B_{1} \Delta x_{\mathrm{Off}} / R+B_{2}\left(\Delta x_{\mathrm{Off}}\right)^{2} / R^{2}
$$

Multipole shifts $\Delta x_{\mathrm{Off}}$ in Tab. 2, column 7, have been determined from pole profile geometry, taken from the OPERA model of the dipoles

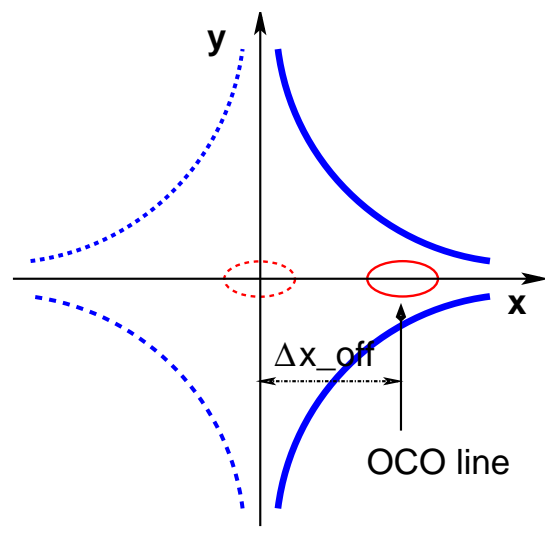

Figure 15: Off-centering of the beam (on the OCO line) in the off-centered multipole model.

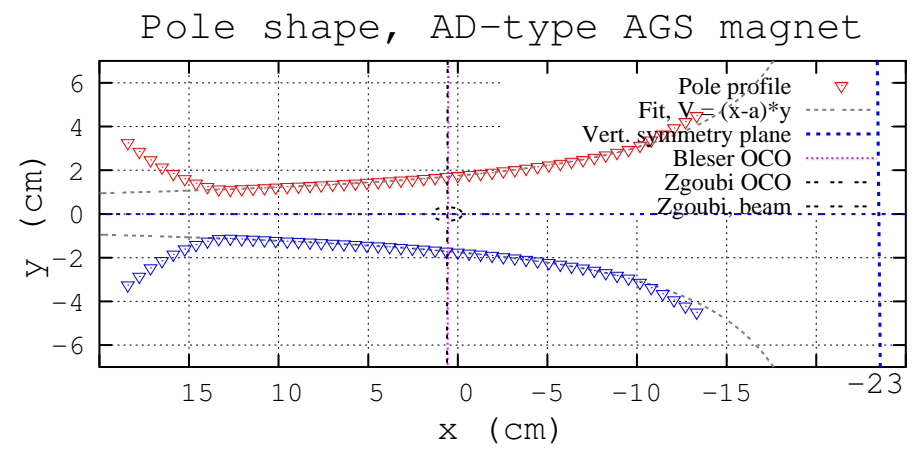

Figure 16: Pole profile in the OPERA model of AGS BD- and ADtype dipole.

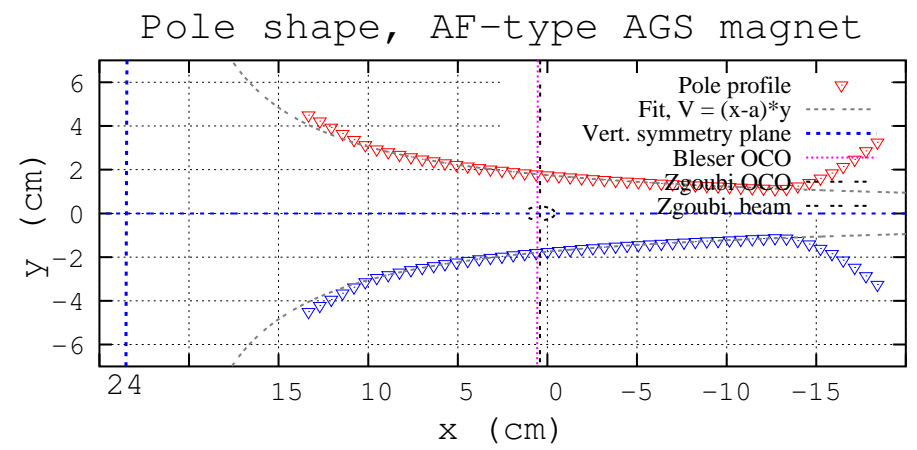

Figure 17: Pole profile in the OPERA model of AGS BF- and AF-type dipole.

(Fig. 16, 17) : the virtual multipole axis is obtained by matching the profile by a potential of the form

$$
V_{2}(x, y)=\left(x-\Delta x_{\mathrm{Off}}\right) y
$$

(neglecting the sextupole component, of little effect). The origin of the referential for $V(x, y)$ is taken at Bleser's 'socket line', $x=0$ in figs. 16, 17. This yields, from Zgoubi closed orbit to Bleser's OCO [44, 43], a residual $-0.31 \mathrm{~mm}$ (outward) in long D-type dipoles (fig. 16), $-1.45 \mathrm{~mm}$ outward in long F-type dipoles (fig. 17). The difference in $\Delta x_{\mathrm{Off}}$ between long and short F-type (resp. D-type) is $1.77 \mathrm{~mm}$ (resp. $1.42 \mathrm{~mm}$ ), close enough to Bleser's value of $1.63 \mathrm{~mm}$ for both. Ring parameters resulting from the off-centered multipole model are shown in Tab. 3, column 4. The beam in the AGS is circulating counterclockwise, dispersion is negative ( $x$ axis is oriented toward ring center, to the left in Figs. 16, 17). The super-period geometry is sketched in Fig. 19.

In that process of finding the offset $\Delta x_{\mathrm{Off}}$ that yield the expected orbit deviation in each one of the 6 types of main magnets, the $K 1$ index is also adjusted, to ensure transport matrices identical to the MAD model ones. Eventually, a difference with MAD, rigidity-dependent, is observed in the tune values, for instance $\delta Q_{x} \approx 0.03, \delta Q_{y} \approx 0.06$ at $G \gamma=45.5$, Tab. 3, col. 4, this is still to be understood.

In this model in its present state a small residual orbit is left (it could be better cancelled with residual adjustment of $\Delta x_{\mathrm{Off}}$ values), it is displayed in Fig. 18.

Finally, this 'off-centered' model has not been exploited up to now, preference has been given to the 'centered' one as it agrees with MAD data and this was deemed useful in a preliminary approach to the AGS. Exploring the former, which is based on a better model of the AGS main magnet, remains to be undertaken.

\section{B.2.2 AGSMM}

The procedure AGSMM in Zgoubi has been derived from MULTIPOL to specifically simulate the AGS main magnet. It uses the same field model, App. B.2, p. 19, and takes its magnetic field and indices $K 1(p)$ (quadrupole), $K 2(p)$ (sextupole), from built-in transfer functions $\mathrm{K} 1-\mathrm{AD},-\mathrm{AF},-\mathrm{BD},-\mathrm{BF},-\mathrm{CD},-\mathrm{CF}$ and $\mathrm{K} 2-\mathrm{AD},-\mathrm{AF},-\mathrm{BD},-\mathrm{BF},-\mathrm{CD}$, -CF (Eqs. 10, 11). Fields and indices are therefore not part of the input data in AGSMM, Tab. 4, by contrast with MULTIPOL. Neither is the length, since it is known for each one of the six types of dipoles, each one is identified in zgoubi.dat by the label that follows the keyword AGSMM ('A01BF' in the present case). The complete structure of the AGSMM data list in zgoubi.dat is shown in Tab. 4. 
Table 3: AGS parameters in the two multipole models, centered (col.3) and offcentered (col.4), bare lattice, no snakes, $G \gamma=45.5$, hard-edged magnets. Data from the MADX model are shown for comparison (col. 2). Orbit and optical functions (last 4 rows) are taken at entrance of A01BF. Note that in the centered model the orbit is quasi-zero, whereas the residual orbit in the off-centered model is $\approx 1 \mathrm{~mm}$ peak-to-peak (Fig. 18).

\begin{tabular}{lccc}
\hline & MAD & \multicolumn{2}{c}{ Zgoubi } \\
& & $\begin{array}{c}\text { centered } \\
\text { multipole }\end{array}$ & $\begin{array}{c}\text { off-centered } \\
\text { multipole }\end{array}$ \\
Orbit length (m) & 807.0913 & 807.0913 & 807.0894 \\
Qx & 8.68727 & {$[8] .68747$} & {$[8] .65178$} \\
Qy & 8.73440 & {$[8] .73463$} & {$[8] .67572$} \\
Q'x & -23.28 & -23.27 & -23.44 \\
Q'y & 3.935 & 3.935 & 4.307 \\
$\alpha$ & 0.01408 & 0.01408 & 0.01419 \\
Periodic functions at 'Begin SuperA' & & \\
$x_{\text {co }}, x_{c o}^{\prime}(\mathrm{mm}, \mathrm{mrad})$ & 0,0 & $\approx 0,0$ & $0.43,0.06$ \\
$\beta_{x}, \beta_{y}(\mathrm{~m})$ & $19.77,11.77$ & $19.77,11.77$ & $19.86,11.86$ \\
$\alpha_{x}, \alpha_{y}$ & $-1.57,1.03$ & $-1.57,1.03$ & $-1.57,1.03$ \\
$D_{x}, D_{x}^{\prime}(\mathrm{m}, \mathrm{rad})$ & $-2.06,-0.147$ & $-2.05,-0.147$ & $-2.09,-0.147$ \\
\multicolumn{3}{r}{} \\
\hline
\end{tabular}

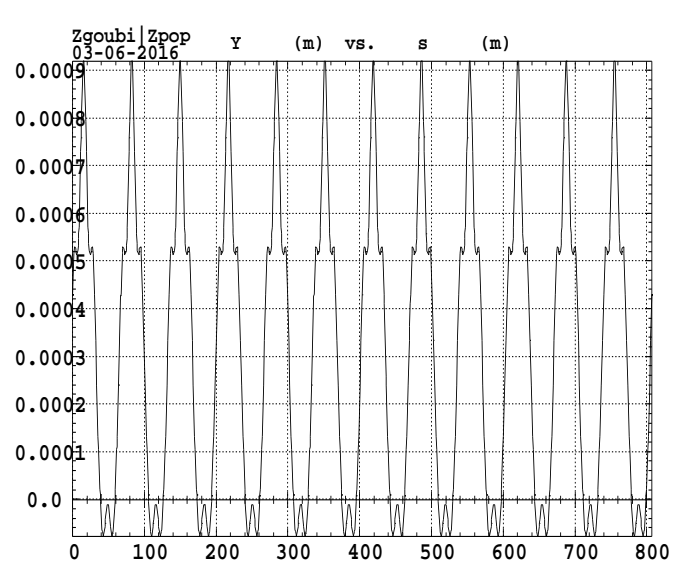

Figure 18: Residual $\approx 1 \mathrm{~mm}$ peak-to-peak orbit in the off-centered multipole model.

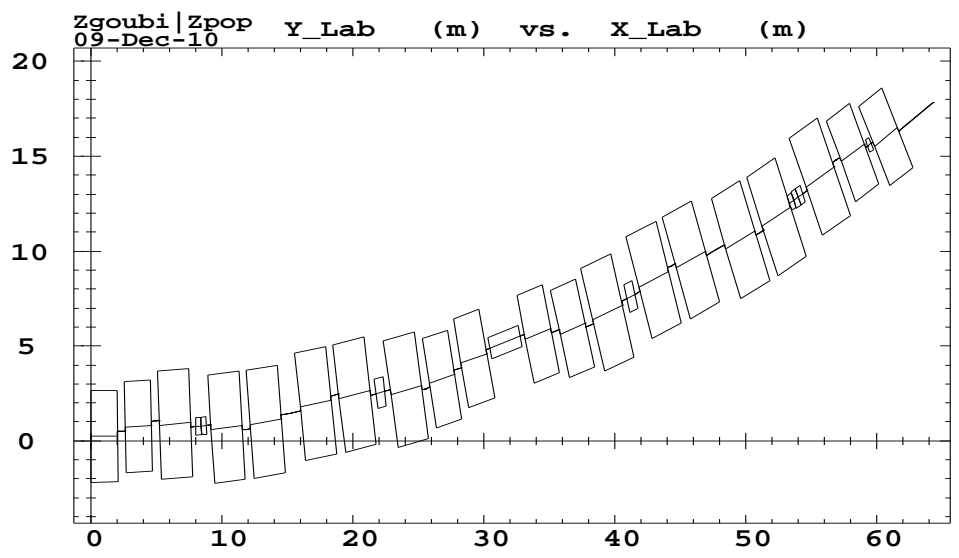

Figure 19: An AGS super-period in Zgoubi, in the off-centered multipole model. The origin of the super-period is at $(0,0)$ on Zgoubi OCO line. The beam circulates counter-clockwise.

AGSMM by default uses the centered multipole model, however a switch might be introduced if desired, so to allow changing to the off-centered multipole model.

The optics of the 'bare AGS' (the AGS with the sole main magnets) can be compared to the MADX model, it comes out very good, at all rigidities, as displayed in Fig. 20. Further comparisons with the bare model in zgoubi and parameterization of the AGS tunes and B.2.3naticitifise catelas foreseen, based on [47].

Measured field maps (see App. B.3) have allowed producing end-field fall-offs in Enge model (Eq. 8). These fringe fields can be accounted for in both AGSMM and MULTIPOL based main magnet models. The evaluation of their impact on beam and spin dynamics has not been especially investigated, so far.

\section{B.2.4 Tracking accuracy}

To conclude with AGSMM and MULTIPOL models in the AGS lattice, the figures below display sample phase space and spin tracking over an acceleration cycle from 9 to $32 \mathrm{GeV}$ (above $8.5 \mathrm{GeV}$, so to avoid $\gamma_{t r}$ gymnastics for simplicity), at a rate of $150 \mathrm{keV} / \mathrm{turn}$, for a particle launched with $\epsilon_{x} \approx \epsilon_{y} \approx 0.2 \mu$ m normalized, using an integration step size $\Delta s=1 \mathrm{~cm}$ (in Eq. 2 for motion, Eq. 5 for spin - p. 18). It can be checked that the damping does satisfy $\beta \gamma \epsilon=C^{s t}$, whereas spin resonance crossings, in a row, do fulfill $P_{f} / P_{i}=2 \exp \left(-\pi \epsilon_{k}^{2} / 2 a\right)-1\left(\epsilon_{k}\right.$ is the resonance strength and can be computed from thin lens model, details and values can be found in [3]; $a$ is the crossing speed). 
Table 4: Structure of AGSMM data list in zgoubi.dat. Line (1) below allows introducing defects on length, field or field indices, with respect to the transfer functions (Eqs. 10,11). Line (2) accounts for the existence of back-leg windings (1 or 2), as occurs in some of the AGS main magnets, and for each winding, its number of turns and intensity. Line (9) allows various misalignments, it can be combined with roll angles of any of the 3 multipoles, line (7). For the rest, AGSMM data are as for MULTIPOL [28].

AGSMM A01BF

0. 0. 0. 0. 0 .

2100.10.

10.04 .00 .8 0. 0. 0. 0. 0. 0. 0. 0 .

$30.262424 .10587-1.03861$ 0. 0. 0.0 .

10.04 .00 .8 0. 0. 0. 0. 0. 0. 0. 0.

$30.262424 .10587-1.038610 .0 .0 .0$.

0. 0.0 .

2.0 Dip MM_A01BF

4 0. 0. 0. 0. 0 .
! (1) dL/L, gap, db/b, dK1/K1, dK2/K2,

! (2) Number of back-leg windings, N, I, N, I,

! (3) Entrance fringe field extent

! (4) Enge coefficients

! (5) Exit fringe field extent

! (6) Enge coefficients

! (7) Roll angle of multipoles

! (8) Integration step size

! (9) Misalignment: X-shft, Y-shft, Z-rot, Z-shft, Y-rot

ZGOUBI MODEL, FROM SNAP RAMPS - Au, 10 June 2011, 10:45

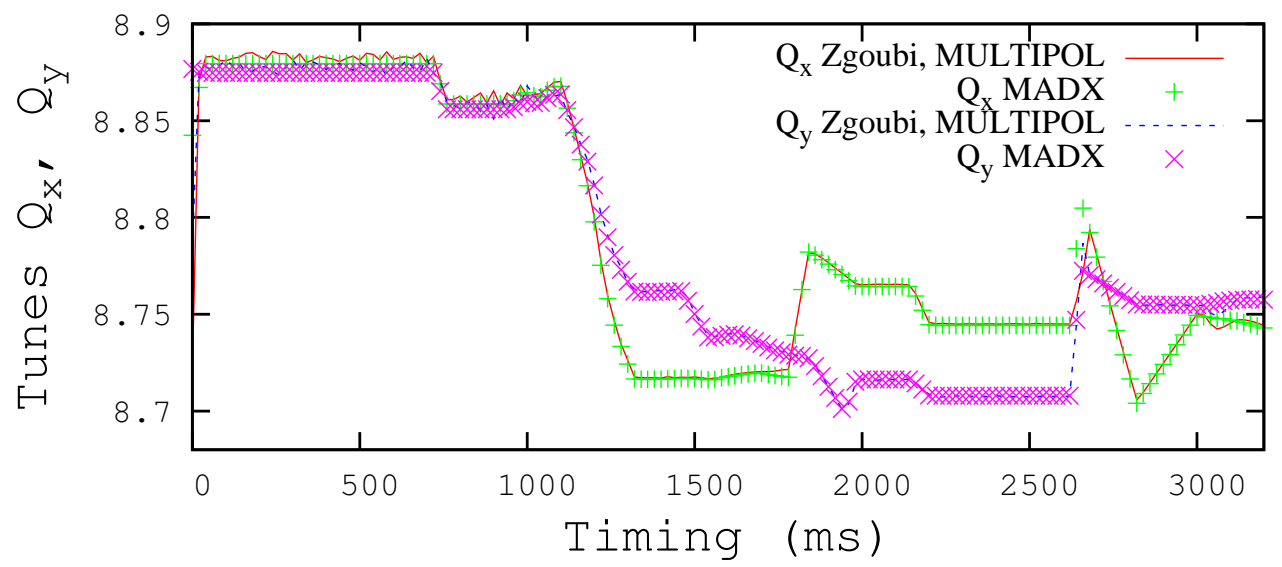

Figure 20: Tunes over AGS cycle from Zgoubi, compared with MADX ones, a case of Gold ion optics settings taken from archived AGS snapramp data (2011).
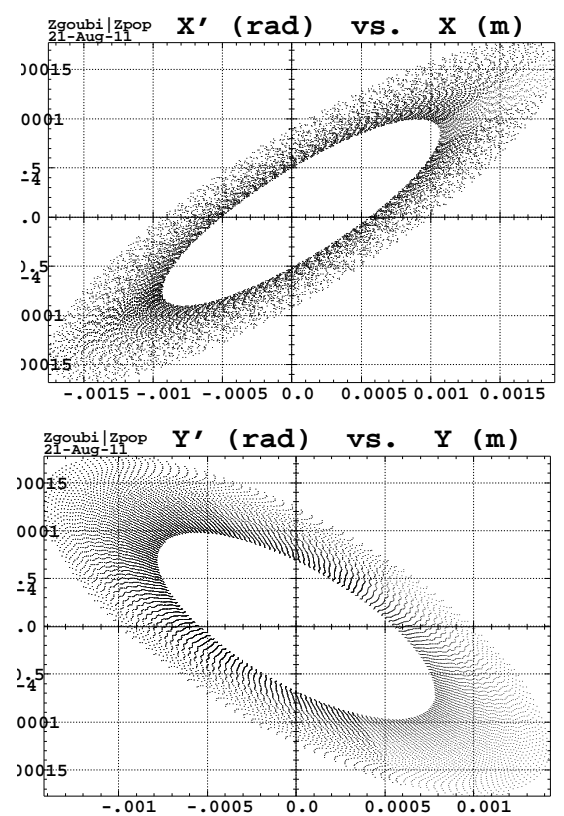

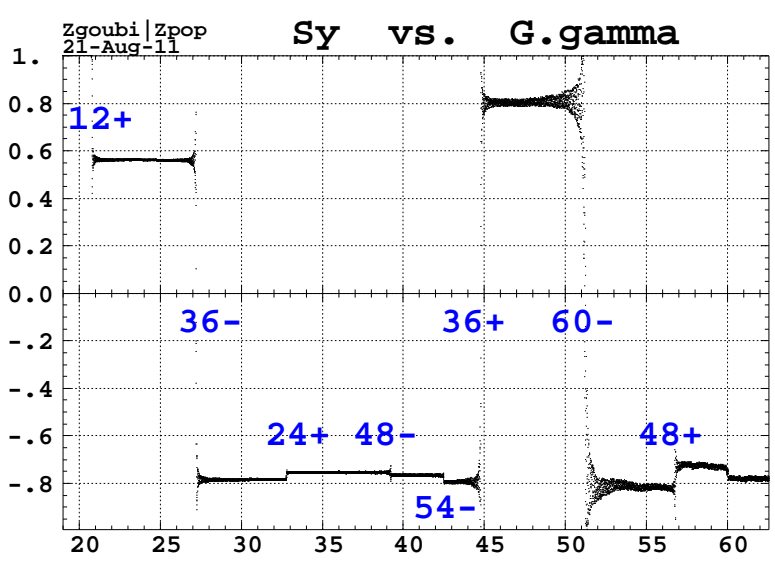

Figure 22: Crossing of intrinsic resonances, in a row, from 9 to $32 \mathrm{GeV}$.

Figure 21: Damped phase space motion, from 9 to $32 \mathrm{GeV}$, horizontal (left) and vertical (right). Both satisfy $\beta \gamma \epsilon=C^{s t}$. 


\section{B.3 AGS main magnet from measured field maps}

We dispose of two series of measured field maps, half the magnet length (Fig. 23), vertical field component in the median plane, for respectively the A- and C-type magnets, 13 currents each, ranging in 15 - 5850 Ampères. A B-type series is derived from the A-type one by shortening the central region of the field map. Reverse focusing for all three series, cf. Fig. 14, is obtained by $x$-flipping the field data. A complete map is obtained by $(x, z)$ vertical plane symmetry.

\section{B.3.1 New, interpolated field maps}

The mesh size in all these maps is $\Delta x=0.1$ inch transverse and, longitudinally, $\Delta z=0.25$ inch in high $d B / d z$ regions, 1 inch in flat field regions (end and body). However Zgoubi prefers uniform mesh, so these maps have been converted to new sets with identical node distances over the all mesh, namely, $\Delta x=0.1$ inch, $\Delta z=0.25$ inch. From a practical point of view, the old mesh geometry has been maintained in the new field maps, and the missing nodes have been added.

The field at all nodes, existing and new, in the new maps, has been interpolated using a second degree polynomial and a $3 \times 3$ interpolation grid, thus ensuring a smoothing of the measured data, which does not cause noticeable change at the old nodes while ensuring a smoothing which is favorable to long-term tracking accuracy. For instance in the case of the $3550 \mathrm{Amp}$, A-type field map, during these interpolation processes, the maximum difference in field value between the old nodes and their new counterparts was found to be less than 0.7 Gauss. General results so obtained over the 30 measured field maps available are shown in Fig. 24. As an illustration an AGS D/F cell is reconstructed and yields the field in Fig. 25.

Some residual oscillation of the data is visible at magnet ends, amplified by $z=5 \mathrm{~cm}$ off-mid plane extrapolation, in Fig. 26 (very lowfield region, a marginal contribution to overall deviation). How much that affects the large amplitude particle and spin dynamics, and whether additional smoothing would be useful or not at field fall-offs, this still remains to be investigated. Anyway stable tracking over thousands of turns is obtained using these maps as they are (see Fig. 30). A great advantage of these measured field maps is that they provide a very realistic model, in particular for the computation of paraxial quantities as orbit, spin $\vec{n}_{0}$ vector, tunes, chromaticities, as this is a matter of 1-turn mapping.

AGS field map model is installed in ZgoubiFromSnaprampCmd, it still has to be exploited.

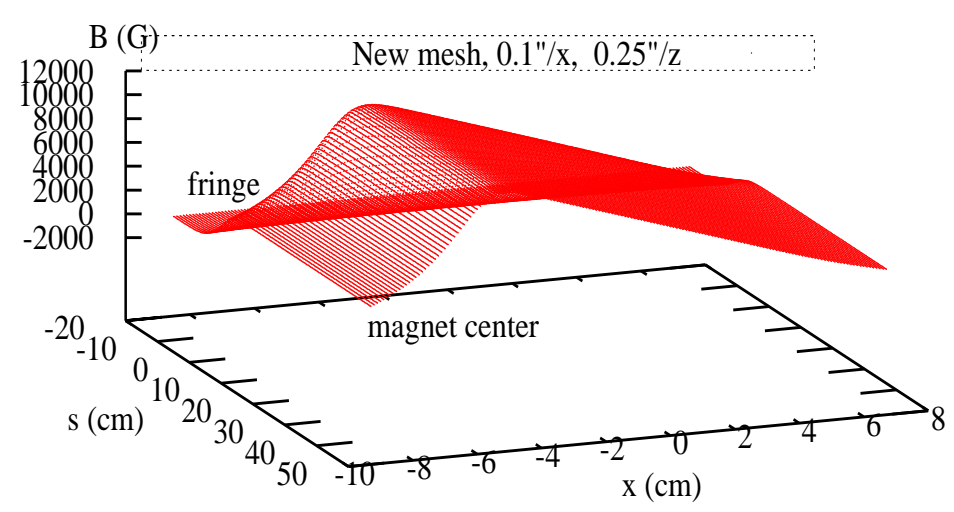

Figure 23: Typical measured field map data. Coordinate $\mathrm{s}$ is longitudinal, $\mathrm{x}$ is transverse horizontal.

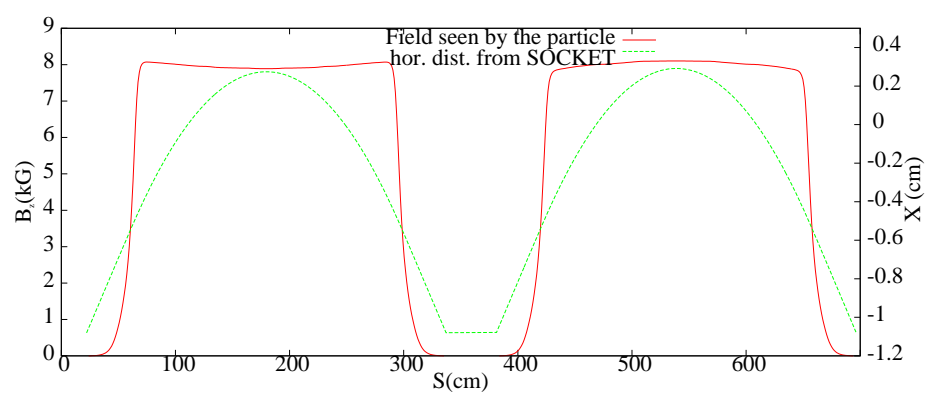

Figure 25: Typical field along the OCO in a D/F map doublet, and distance to the socket line.

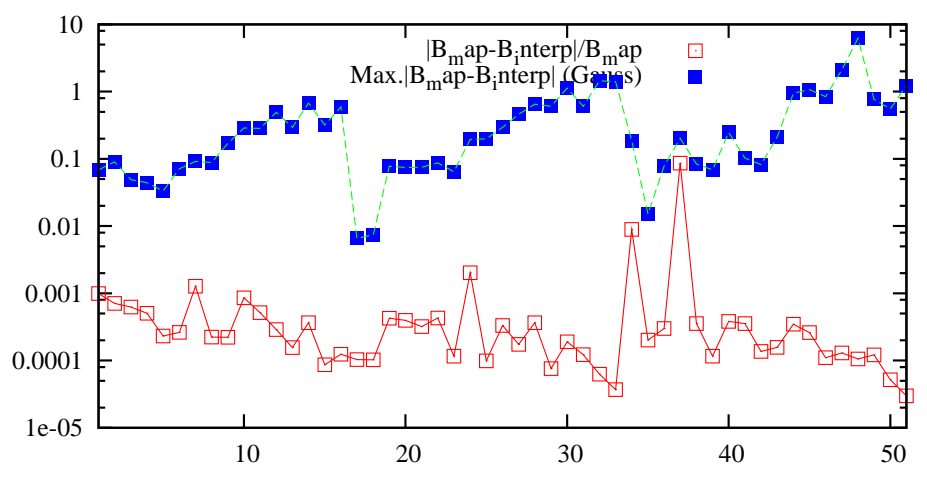

Figure 24: Average relative difference, and maximum difference in Gauss, over all mesh nodes, over the 30 available field maps.

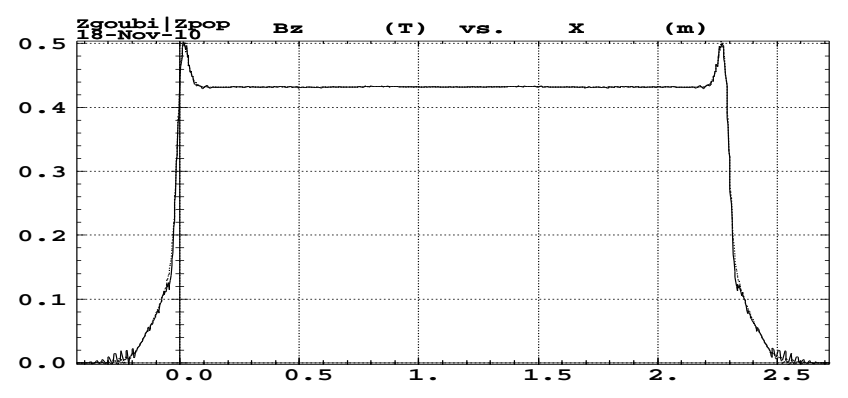

Figure 26: Vertical field along an arc parallel to the OCO, $5 \mathrm{~cm}$ off median plane, as obtained by extrapolation from the mid-plane data.

\section{B.3.2 K1 and K2 polynomials}

These field maps have been used to determine, based on Zgoubi ray-tracing, the quadrupole strengths $K 1(I)$ and sextupole strengths $K 2(I)$ [49], see results for $K 1(I)$ displayed in Figs. 27, together with polynomials as found in reference [45], for reference (similar, close 
polynomial coefficients, are obtained for $K 2(I))$. The correspondence between magnet current, $I$, and reference momentum $p$ in the ring,

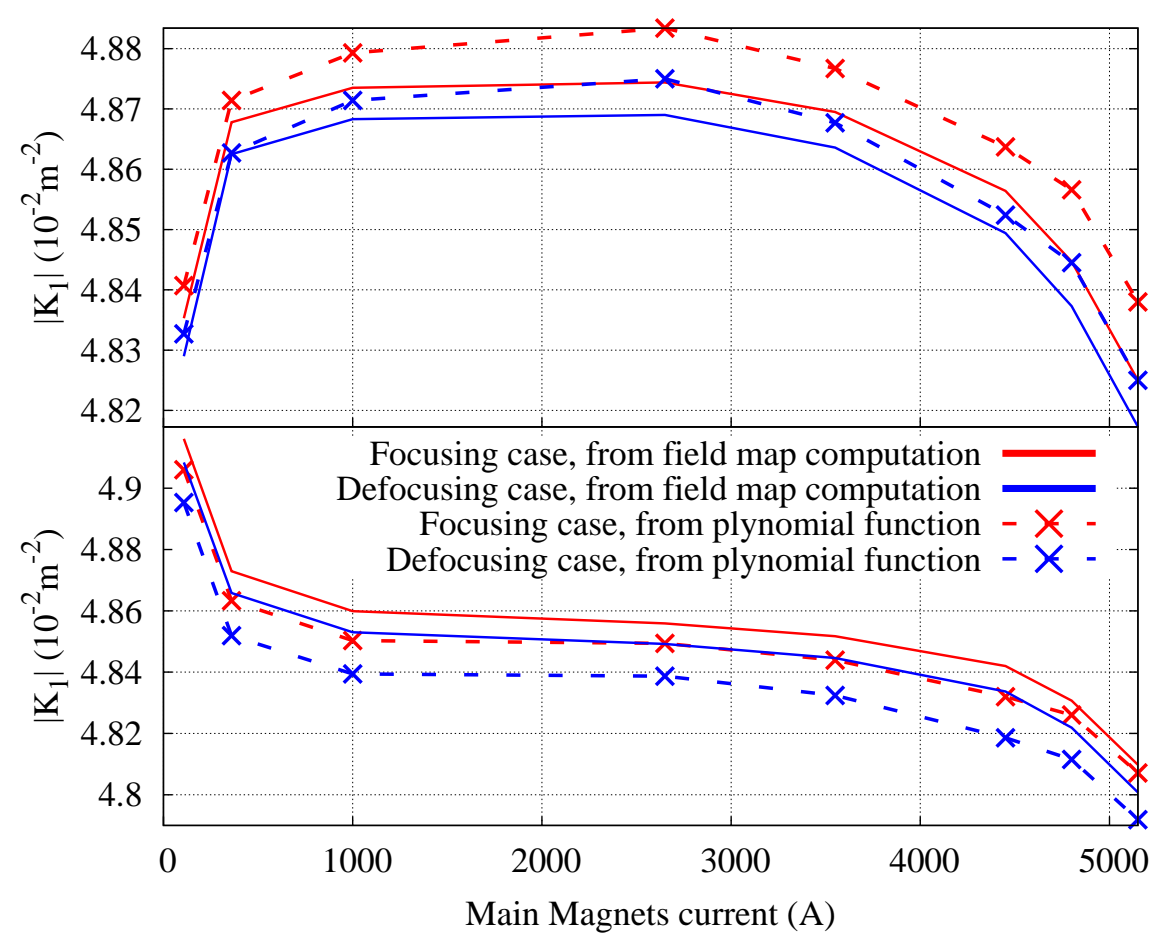

Figure 27: $K_{1}$ versus current in (top) A- and (bottom) C-type magnets. Solid lines are the strengths from Ref. [45], dashed lines are data obtained from Zgoubi raytracing, the difference is marginal. Similarly, close polynomials are obtained for $K_{2}(I)$.

can also be found in Ref. [46], thus allowing matching $K 1(p)$ and $K 2(p)$ with polynomials of Eqs. 10, 11. Differences observed between the original $K 1(p)$ and $K 2(p)$ series and those obtained from Zgoubi ray-tracing [11, Tabs. B1, B2 in App. B] are marginal.

\section{B.3.3 Fringe field coefficients}

AGSMM and MULTIPOL support fringe fields, based on Enge model (Eq. 8). Enge Coefficients have been drawn from the measured field maps, as follows.

The effective field boundaries in the AGS main magnets are located 2 inches from the iron end, outward, following Bleser, Fig. 12 [44, 43], resulting in effective lengths of 90+4 inches, long dipole (A and C types, Fig. 14), and 75+4 inches, short dipole (B type). The zero of the $\frac{1}{2}$-magnet measured field maps coincides with the iron edge.

The fringe field study reported here is for the b3550.mpa B-type magnet field map, current $3550 \mathrm{Amp}$, other field maps and currents do not differ significantly, so far as low order optics is concerned.

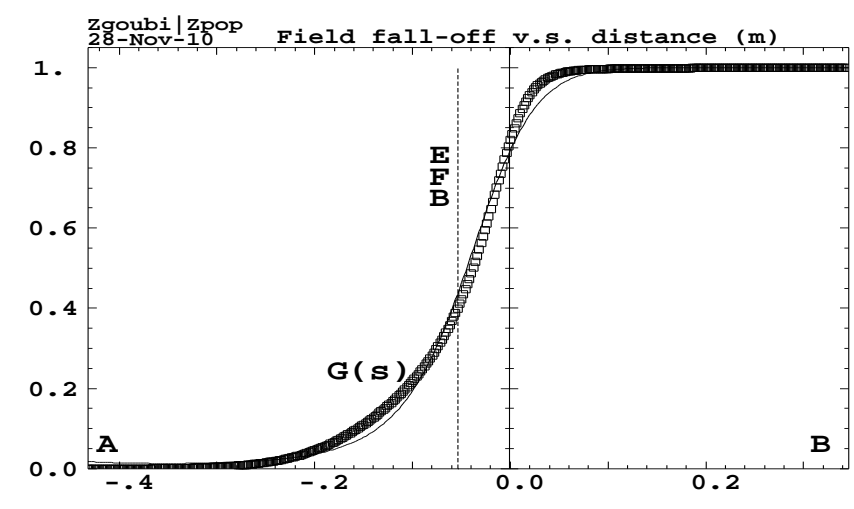

Figure 28: Field fall-off from b3550.mpa measured field map (squares) and its interpolation with the Enge exponential model (solid line). The EFB (vertical line) as delivered by the matching falls 2.13 inches from the origin of the field map (which is at the ion), in good accord with Bleser's 2" (Fig. 12). 
Based on the definition of the EFB, namely,

$$
\int_{A}^{E F B} G(s) d s=\int_{E F B}^{B} d s-\int_{E F B}^{B} G(s) d s \Rightarrow d_{E F B}=d_{B}-\int_{A}^{B} G(s) d s
$$

and computing $\int_{A}^{B} G(s) d s$ numerically from field map data, one gets the distance

$$
d_{E F B}=-5.40 \mathrm{~cm}=-2.13 \text { inches }
$$

from the EFB to the origin in the map frame, very close to Bleser's 2 inches from the iron to the EFB (Figs. 12, 13, p. 19).

The figure shows the interpolation of the fall-off from b3550.mpa field map with Enge exponential model limited here to three coefficients,

$$
C_{0}=0.26242, C_{1}=4.10587, C_{2}=-1.03861
$$

Comparisons of the AGS model using field maps or using AGSMM with these three $C_{0}-C_{2}$ remain to be undertaken.

\section{B.3.4 Tracking in field maps, thousands of turns}

Sample multiturn tracking results are shown in Figs. 29, 30. The stability limit in these transverse phase spaces stems from limited field map size (over-extrapolation of field values out of the map). It is clear however that the field map extent is large enough to get reasonable tracking quality, over a few 1000 turns range, far beyond beam occupation region. These tracking samples also show that the accuracy on
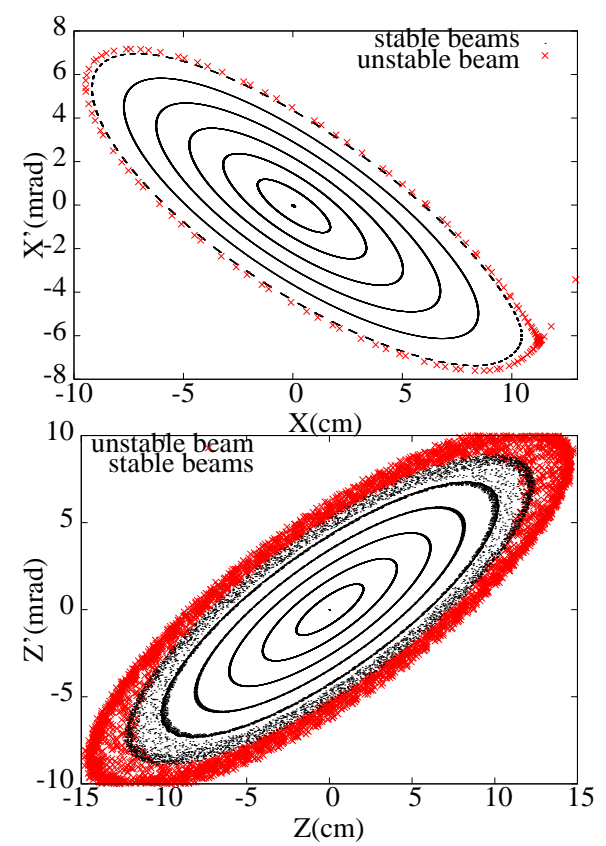

Figure 29: Numerical stability limits, horizontal (top) and vertical phase space (bottom).

motion computation using measured field maps is reasonable enough to yield first order machine parameters, chromaticities. Spin motion and resonance effects also are within reach using measured field maps, Fig. 30, as crossing of a $G \gamma=$ integer or $G \gamma \pm \nu_{y}=$ integer resonance takes $3 \sim 4$ thousand turns.

\section{B.4 AGS main magnet from OPERA field maps}

3-D OPERA field maps of the AGS main magnets are available [51]. They have been archived at

$$
\text { /rap/lattice_tools/zgoubi/AgsZgoubiModel/mainMagnetFieldMaps/ }
$$

Their handling is as discussed in the previous case of measured field maps, App. B.3.

A better motion computation accuracy ('symplecticity') is expected as the mesh is tighter, and field is smooth by contrast with measured maps. OPERA maps should allow beam and polarization transport through the entire acceleration cycle, about 150000 turns. As a matter of fact,

- cold and warm snake 3-D OPERA maps are routinely used in the AGS model, and in studies requiring full cycle, 150000 turn simulations [11],

- it has been shown in various other occasions that computed field maps do give access to accurate long-term tracking, even in highly non-linear context, as tens of thousand of turns in FFAG magnets using 3-D OPERA maps [52, 53]. 


\section{B.5 AGSQUAD}

The procedure AGSQUAD in Zgoubi has been derived from MULTIPOL to specifically simulate the AGS quadrupoles, and in particular multiple windings with independent power supplies, up to three. It uses the potential model $V_{2}(s, x, y)$, Eq. 7, p. 19. Whereas MULTIPOL requires fields at pole tip, AGSQUAD requires winding currents. The field is computed from the ampere-turns in the coils using transfer functions that have been copied from the MADX model software and have their origin in reference [45]. The multipole winding procedure allows overlapping of quadrupole families. As an illustration, this is the case of the double-winding quadrupole QP_B03, Tab. 5, which belongs in both the tuning quadrupole family and the cold snake compensation quadrupoles family.

Table 5: Structure of AGSQUAD data list in zgoubi.dat. QP_B03 is an instance of a multiple-winding quadrupole, two in its case, with current values I1=-296 Amp, I2=9.1187916 Amp, row 3 in the list.

\begin{tabular}{|c|c|}
\hline AGSQUAD & ! label \\
\hline \multicolumn{2}{|l|}{0. Quad } \\
\hline 0. 0. 0.0.0.0.0.0.0.0.0. & ! hard edge entrance \\
\hline $6.11226 .2671-1.49823 .5882-2.12091 .723$ & ! entrance Enge coefficients (unused, here) \\
\hline 0.0.0.0.0.0.0.0.0.0.0. & ! hard edge exit \\
\hline $6.11226 .2671-1.49823 .5882-2.12091 .723$ & ! exit Enge coefficients (unused) \\
\hline 0.0 .0 .0 .0 .0 .0 .0 .0 .0 & \\
\hline \#10|10|10 Quad QP_B03 & ! number of steps in in-fringe|body|out-fringe \\
\hline 10.0 .0 & \\
\hline
\end{tabular}

\section{B.6 Helical snakes}

The two AGS helical snakes are simulated using their 3-D OPERA field map.

The OCO at the snake requires two considerations : firstly, it has to be moved during acceleration so that the helical trajectory across the snake be maintained centered on the snake axis ; secondly, the orbit defect that snakes introduce has to be corrected. In real life, it is only corrected for the cold snake (the warm snake is weaker). However, in most simulations so far it has been considered desirable to work with zero orbit around the ring (but for the two local snake bumps), for that reason the orbit effect of the two snakes is fully compensated, locally, Fig. 31. On the other hand, from the point of view ease of manipulation of the model, and in order to allow flexibility in optics and polarization studies, it is desirable that this local orbit bump and residual orbit correction do not encompass the snake-compensation and other tuning quadrupoles which are located close upstream and downstream of the helix. Doing so presents the advantage that a change in quadrupole settings will not affect the orbit bumps. However a trick is used instead of introducing strong local bump dipoles at snake ends (this would noticeably affect the time-of-flight as well as spin motion), namely, (i) the snake field map is moved horizontally during acceleration, by an amount which is momentum dependent and ensures the centering of the helix on the snake axis, (ii) the remaining, small, orbit defect is corrected locally using weak correctors located right upstream and downstream of the snake. Being weak, these residual orbit corrections have negligible effect on orbit length (revolution frequency), and on spin motion.

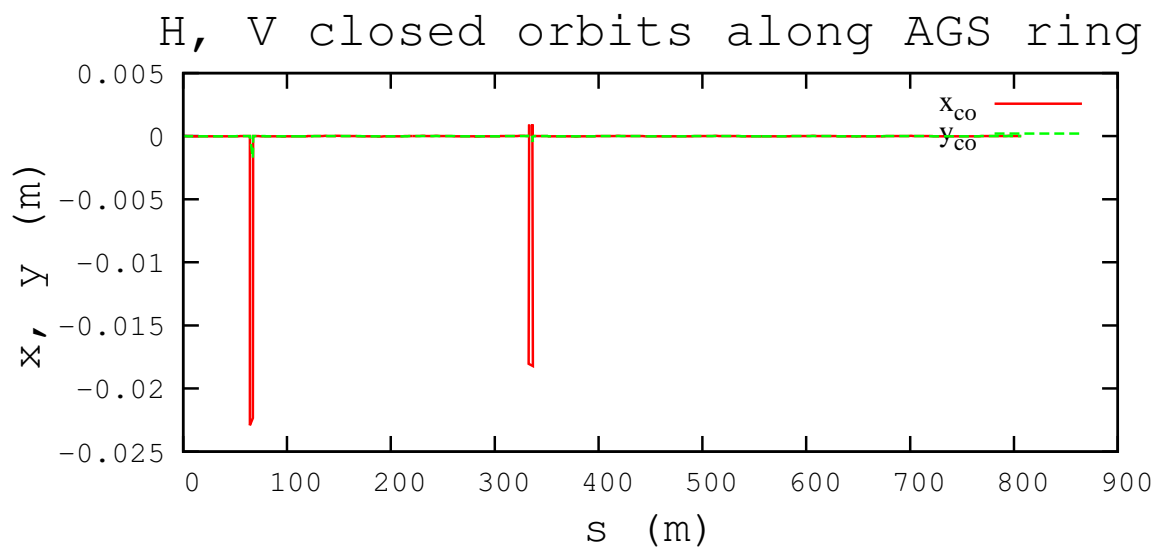

Figure 31: Zero $\mathrm{x}$ and $\mathrm{y}$ closed orbits around the AGS ring with local orbit bumps at the cold (left) and warm (right) snakes (including a very small, yet non-zero y-bump), in the standard AGS Zgoubi model. Here at injection, $G \gamma=4.5$. The bumps decrease to less than $2 \mathrm{~mm}$ at extraction, $G \gamma=45.5$ (snakes operate at constant field). 


\section{B.7 Symplecticity tests}

Long-term tracking is required in assessing depolarizing effects in AGS, involving large motion invariants $\epsilon_{x}, \epsilon_{y}$. On the other hand, Zgoubi does not use any simplectization method, accuracy of numerical integration is controlled essentially via step size, and possibly Taylor series extents in $\vec{R}, \vec{u}, \vec{S}$ in the integrator (App. A). This imposes keeping an eye on symplecticity of motion - and spin - tracking.

A model of the AGS obtained from 10 June 2011 ppmUser1 snap ramps has been built for that, using ZgoubiFromSnaprampCmd, snakes and skew sextupoles are off, chromaticity sextupoles are on. The centered-multipole model (App. B.2.1, p. 20) is used in this model.

General parameters of the ring, from Zgoubi with snap ramp settings, are as follows ;

$\begin{array}{lcc}\text { circumference } & (\mathrm{m}) & 807.0909 \\ \text { transition } \gamma & & 8.472 \\ \text { tunes x, y } & & 8.721,8.785 \\ \text { chromaticities x, y } & & -14.8,-2.16\end{array}$

A 150000 turns acceleration cycle is performed, at a rate $d E=150 \mathrm{kV} / \mathrm{turn}$, starting from $9 \mathrm{GeV}$ (so to avoid transition-gamma optics region, not relevant in the present study) to $31.807 \mathrm{GeV}$. The integration step size is $\Delta s \sim 1 \mathrm{~cm}$ in all optical elements. The tracking is realized for paraxial rays, starting invariants $\epsilon_{x}, \epsilon_{y} \sim$ a few $10^{-8} \pi \mathrm{m}$.rad. Results are shown in figure 32 and Tab. 6 . The agreement between numerical and expected damping is within 1\%) - regardless of possible (small) effects as non-linear coupling, sextupole fields. This is an indication of appropriate quasi-symplectic behavior of the integration in the present numerical conditions.
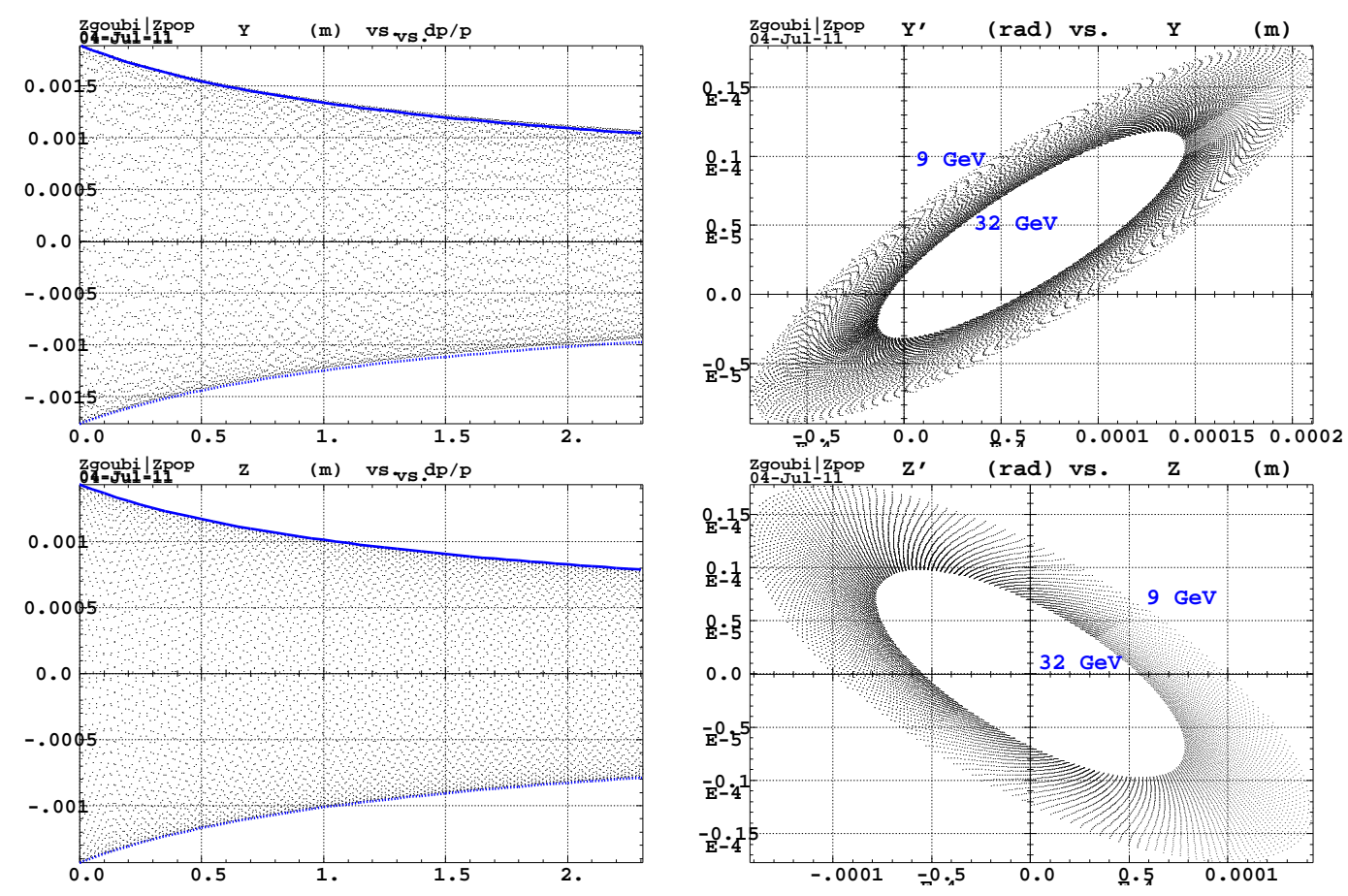

Figure 32: Left column : damped horizontal (top) and vertical (bottom) particle excursions over a 144500 turn acceleration cycle with theoretical envelopes $\hat{x}(0) \times \sqrt{p_{0} / p}$ superimposed (blue curves) for comparison. Right column : the related horizontal (top) and vertical (bottom) phase spaces, showing damped particle motion.
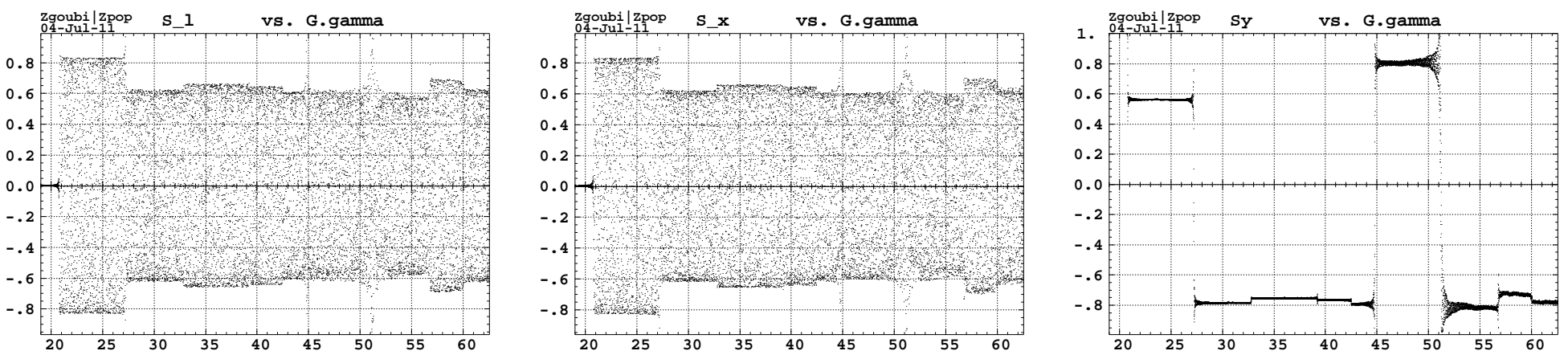

Figure 33: Evolution of the spin components as a function of energy $(G \gamma)$, for starting $\vec{S} \equiv \vec{S}_{y}$, from $9 \mathrm{GeV}$ to $31.807 \mathrm{GeV}$. From left to right : $S_{l}$ (longitudonal), $S_{x}$ (transverse horizontal), $S_{y}$ (vertical). 
Table 6: Damping results, after 90530 and 144500 AGS turns. Both $\epsilon_{x 0} / \epsilon_{x}$ (rwo 5) and $\epsilon_{y_{0}} / \epsilon_{y}$ (row 7) appear to closely satisfy $B \rho$ damping (row 3). Note that the lattice is non-linear (main contributions : lattice sextupoles and (weak) $K_{2}$ component in main magnets), which may contribute to the observed departing from conservation of normalized concentration ellipse surface, to be determined.

\begin{tabular}{lcccc}
\hline & & & & \\
turn \# & & 1 & 90530 & 144500 \\
kinetic E & $(\mathrm{GeV})$ & 9 & $\mathrm{~m}(45.5-1) / \mathrm{G}$ & 31.807 \\
$B \rho / B \rho_{0}$ & & 1 & 2.445 & 3.308 \\
$r m s \epsilon_{x}$ & $\left(10^{-8} \pi \mathrm{m} . \mathrm{rad}\right)$ & 8.431 & 3.429 & 2.528 \\
$\epsilon_{x 0} / \epsilon_{x}$ & & 1 & 2.459 & 3.335 \\
$r m s \epsilon_{y}$ & $\left(10^{-10} \pi \mathrm{m} . \mathrm{rad}\right)$ & 8.908 & 3.614 & 2.661 \\
$\epsilon_{y 0} / \epsilon_{y}$ & & 1 & 2.465 & 3.348 \\
\hline
\end{tabular}

Spin behavior is shown in Fig. 33. The departure of $\left(S_{x}^{2}+S_{y}^{2}+S_{z}^{2}\right)$ from 1 over the 144500 turns is below computer precision.

These results indicate that, with careful installation of Zgoubi input data it is possible to realize full acceleration throughout AGS cycle with satisfactory tracking accuracy of both motion and spin. This example does not include the snakes in AGS lattice, however extensive tests and tracking simulations have been performed [11, Section 4.4.1.1] [34] which assessed the accuracy of the tracking in presence of the snakes, and showed convenient Courant invariant behavior over complete $G \gamma: 4.5 \longrightarrow 45.5$ AGS acceleration cycle.

\section{From AGS snapramps to Zgoubi to the AGS model : functionning of ZgoubiFrom- SnaprampCmd}

This appendix comments on the functioning of ZgoubiFromSnaprampCmd command.

There are two possible ways to specify the input data to ZgoubiFromSnaprampCmd,

- either via an input file, a specimen is given in App. D.1,

- or via arguments in a similar way to MadxFromSnaprampCmd. They are listed in App. D.2.

Input data files to Zgoubi (zgoubi.dat in the following) for a model of the AGS at one or more arbitrary timings are created from an AGS snapramp (of which the address is part of the input data to ZgoubiFromSnaprampCmd), i.e., a set of files (Fig. 34), records of the time dependence (Fig. 35) of all magnet power supplies over the AGS cycle. Conversion from snapramps to magnet fields in zgoubi.dat is taken care of by the 'conversion' procedure, a translation to Fortran of MadxFromSnaprampCmd one [24]. ZgoubiFromSnaprampCmd produces one zgoubi.dat input data file to Zgoubi per timing 'tttt', each one saved in a dedicated folder model.tttt.zgoubi (Fig. 36).

Once zgoubi.dat is available, ZgoubiFromSnaprampCmd runs it and produces a MADX-style twiss file for each timing, zgoubi.TWISS.out, with the very format of MADX one (App. D.4, p. 35). These twiss files are all stored in a single twiss folder (Figs. 36, 37). In addition to these, the machinery produces various other files stored in the model.ttt.zgoubi folders, including

- TWISS_bruteFromSnapramp.res, an early result of Zgoubi execution,

- fitTunesCmd.res : in case a tune match has been requested (as part of ZgoubiFromSnaprampCmd input data), it contains the corresponding results - this is the case that produced Fig. 2, p. 8, a new TWISS.res is produced in that case,

- 'track.dat' : allows 6-D bunch tracking at the particular energy corresponding to timing=tttt. Note that it also allows, with little modifications, to accelerate a 6-D bunch from and to arbitrary timings,

- various plots (as in Fig. 38).

and some other subproducts of the run. These various outcomes are aimed at investigating beam optics and polarization dynamics in close relationship with experimental data taking.

\section{C.1 Magnet models}

Three different models are available for the AGS main magnet, any can be chosen for the AGS model, that is part of the input data to ZgoubiFromSnaprampCmd.

These are (App. B.2.2, p. 21)

(i) AGSMM,

(ii) MULTIPOL,

both in either the centered- or off-centered multipole model (App. B.2.1, p. 20),

(iii) field maps,

- either measured (App. B.3, p. 24)

- or from OPERA simulations (App. B.4, p. 26). 


\begin{tabular}{|c|c|c|c|c|c|c|c|c|c|}
\hline A20_BLW_PS1 & Hchrom_I & Vtune_I & ab12-th & ad09-oct & afo2-th & ag18-th & ail8-tv & ak17-qgt & e17-gd \\
\hline A20 BLW PS2 & Htune $\bar{I}$ & a $16-1 \overline{9}$ blw & ab12-tv & ad12-th & afo2-tv & ag18-tv & aj 02 -th & ak18-th & e19-qf \\
\hline A20 BLW_PS3 & I5HighvoltagePS & a17_quâd & ab18-th & ad12-tv & afo8-th & ag19-oct & aj $02-t v$ & $a k 18-t v$ & f1-qd \\
\hline & I5LowVolt agePS & a 19 quad & ab18-tv & ad15. ipm-tv & afo8-tv & aho2-th & aj 08 -th & al02-th & fieldFromRF \\
\hline$B C_{\text {c cav }}$ & IJ_cav & aa02-th & acO2-th & ad16-oct & af 10-blw & ah02-tv & aj08-tv & alo2-tv & fregFromRF \\
\hline$B C$ cav Phase & IJ cav Phase & $\mathrm{aa02}-\mathrm{tv}$ & $\mathrm{acO} 2-\mathrm{tv}$ & ad18-th & af 12 -th & ah08-th & a j09-oct & al08-th & horAveorbit \\
\hline B_cav & J5Highv̄oltagePS & aa08-th & ac05.ipm-th & ad18-tv & af $12-t v$ & aho8-tv & aj 10-dump & alo8-tv & horHarm8Cos \\
\hline B cav Phase & J5LowVol tagePS & $a a 08-t v$ & acos-th & ad19-oct & af 18 -th & ah12-th & aj 12 -th & al 12 -th & horHarm8Sin \\
\hline BdotFromRF & JK_cav & aa09-oct & ac08-tv & ad20-blw & af $18-t v$ & ah12-tv & aj $12-\mathrm{tv}$ & al12-tv & horHarm9Cos \\
\hline C_cav & JK_cav_Phase & aa12-th & ac12-th & ae02-th & ago2-th & ah18-th & aj 16 -oct & al18-th & horHarm9Sin \\
\hline C_cav_Phase & $\mathrm{KL} \mathrm{cav}$ & aa12-tv & ac12-tv & ae 02 -tv & ago2-tv & ah18-tv & aj 18 -th & al 18-tv & radius \\
\hline $\mathrm{DE}$ cav & $\mathrm{KL}$ cav Phase & aa16-oct & ac17-qgt & ae08-th & ag08-th & aio2-th & aj18-tv & al20-angtrim & summary \\
\hline DE_cav_Phase & K_cav & aa17-qgt & ac18-th & aeo8-tv & ag08-tv & a102-tv & aj 19 -oct & al20-blwang & verAveorbit \\
\hline D cav & K cav Phase & aa18-th & ac18-tv & ae12-th & ag09-oct & aios-th & ak02-th & al20-blwpos & verHarm8Cos \\
\hline D_cav_Phase & MM I & aa18-tv & adO2-th & ae $12-t v$ & ag10-blw & aio8-tv & $a k 02-t v$ & al20-postrim & verHarm8Sin \\
\hline E cav & Q skew_I $(t)$ & $a b 02$-th & ad02-tv & ae15. ipm-tv & ag12-th & ail2-th & ak08-th & b1_quad & verHarm9Cos \\
\hline E cav Phase & Spillservo & $a b 02-t v$ & ad05. ipm-th & ae17-gat & ag12-tv & ai12-tv & ak08-tv & b2-5 blw & verHarm9Sin \\
\hline G09bump & vchrom_I & abo8-th & ado8-th & ae18-th & ag16-oct & ail7-ggt & ak12-th & b3 quad & \\
\hline H11bump & vectorsum & $a b 08-t v$ & ado8-tv & ae18-tv & ag17-qgt & ails-th & ak12-tv & beamcurrent & \\
\hline
\end{tabular}

Figure 34: A specimen snapramp folder.

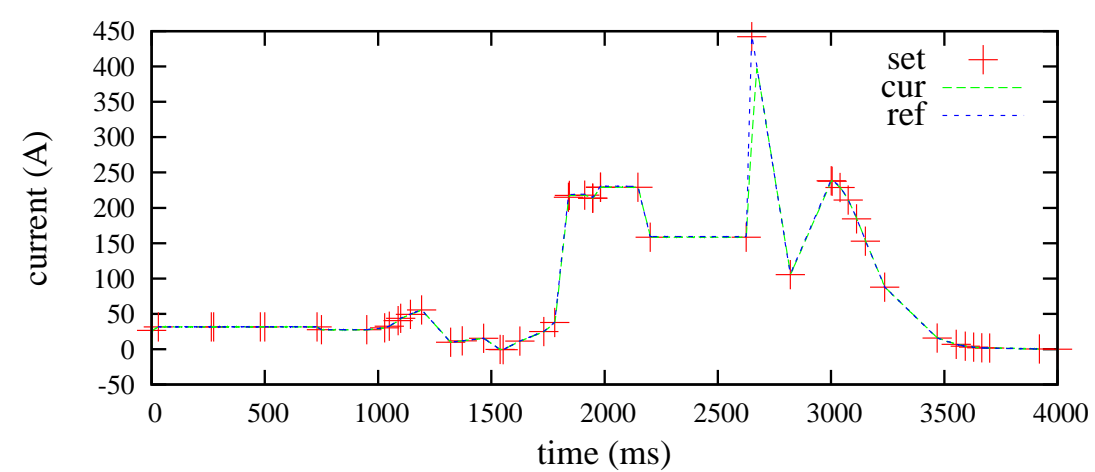

Figure 35: A plot of the content of horizontal quadrupole family power supply file ('Htune_I' file in the snapramp folder, Fig. 34) (three variants of the data are saved : 'set', 'cur' and 'ref'. 'cur' is a measurement of the magnet current, it is the one used by ZgoubiFromSnaprampCmd, it provides data at millisecond intervals).

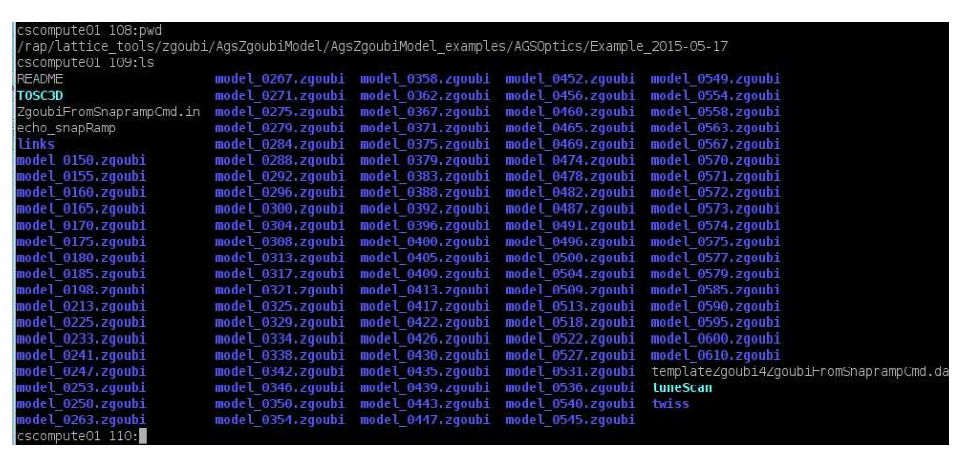

Figure 36: A specimen ZgoubiFromSnaprampCmd model.ttt.zgoubi series, and twiss folder outcome. The content of a model.tttt.zgoubi folder is displayed in Fig. 38.

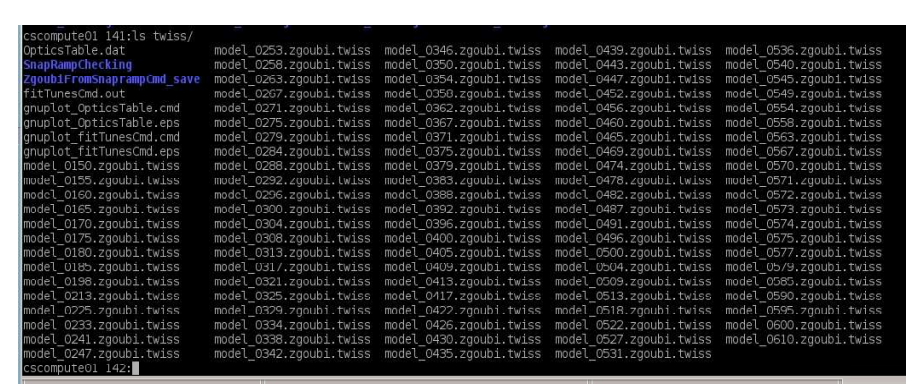

Figure 37: The content of a twiss folder, namely, a series of MADX style twiss files, one per timing (sample in App. D.4), similar to MadxFromsnapramp command outcome.

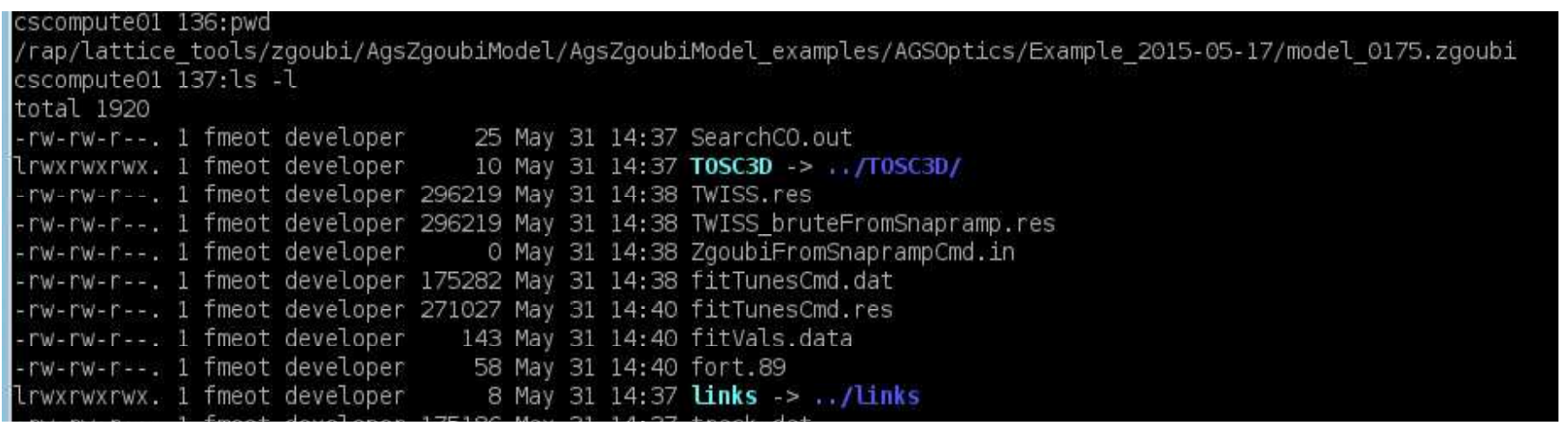

Figure 38: The content of a model.ttt.zgoubi folder. This example is archived at

/rap/lattice_tools/zgoubi/AgsZgoubiModel/AgsZgoubiModel_examples/AGSOptics/Example_2015 - 05 - 17. 
Other magnets found in AGS lattice are

- quadrupoles, simulated using AGSQUAD (App. B.5, p. 27),

- sextupoles, simulated using MULTIPOL,

- partial snakes, simulated using their 3-D field maps and TOSCA procedure (App. B.6, p. 27),

- tune jump quadrupoles, simulated using MULTIPOL.

Correctors are not included in the standard model. Should orbit errors be included, orbit correctors could use MULTIPOL.

\section{C.2 Power supply settings}

The scaling of these magnets to follow the snapramp power supply time evolution is obtained using SCALING (p. 11), a command apparent in the top region of zgoubi.dat and other TWISS.res files in the model.tttt.zgoubi folders (App. D.3). SCALING controls

- the orbit and focusing snake compensation quadrupoles,

- the jump quadrupoles,

- the $\mathrm{F}$ and $\mathrm{D}$ quadrupole families,

- the G10 and H10 extraction bumps (these are set for instance, at timing=1077 ms in Fig. 3, p. 9),

- the $\gamma_{t r}$-quads,

- $d K_{1, F} / K_{1, F}$ and $d K_{1, D} / K_{1, D}$ errors,

and so forth. Main magnets $K_{1}(p)$ and $K_{2}(p)$ indices are determined by the reference momentum (App. B.2.2, p. 21).

App. D.3 gives excerpts of AGS sequence model in Zgoubi, with the elements above and their data list.

\section{C.3 Matching to measured tunes and/or chromaticities}

A possible request to ZgoubiFromSnaprampCmd is the matching of the tunes to external data (e.g., measured). Perturbations $d K_{1} / K_{1}$ and $d K_{2} / K_{2}$ are part of SCALING data list (App. D.3) and are used as the matching variables. There is a similar possibilities regarding chromaticities, using $d K_{2, F} / K_{2, F}$ and $d K_{2, D} / K_{2, D}$ which are also part of SCALING data list.

The accuracy of the agreement between for instance measured on the one hand and AGS model tunes on the other hand (or chromaticities) is controlled via the value of a 'penalty' in Zgoubi's fitting procedure, in the example of Fig. 2 the penalty has been taken small, $3 \times 10^{-7}$, at all timings, so to yield marginal $\left(Q_{\text {measured }}-Q_{\text {model }}\right)$. $\mathrm{dK} 1 \mathrm{~F} / \mathrm{K} 1 \mathrm{~F}$ and $\mathrm{dK} 1 \mathrm{D} / \mathrm{K} 1 \mathrm{D}$ relative changes necessary to get good agreement are usually within $\pm 10^{-3}$ of the unperturbed K1F, K1D values, at all timings, as it is the case in the example of Fig. 2. Such matching over about 100 timings covering the AGS cycle, in that example, takes of the order of a minute, total, to complete - quite compatible with operation timescales (CPU time consumption is discussed in Sec. 3.5). 


\section{I/O files to/from ZgoubiFromSnaprampCmd}

\section{D.1 ZgoubiFromSnaprampCmd.in}

ZgoubiFromSnaprampCmd.in, input data file to ZgoubiFromSnaprampCmd. This is taken from the archived example

$$
\text { /rap/lattice_tools/zgoubi/AgsZgoubiModel/Ags_ZgoubiModel_examples/AGSOptics/Example_2015 - } 05 \text { - } 17
$$

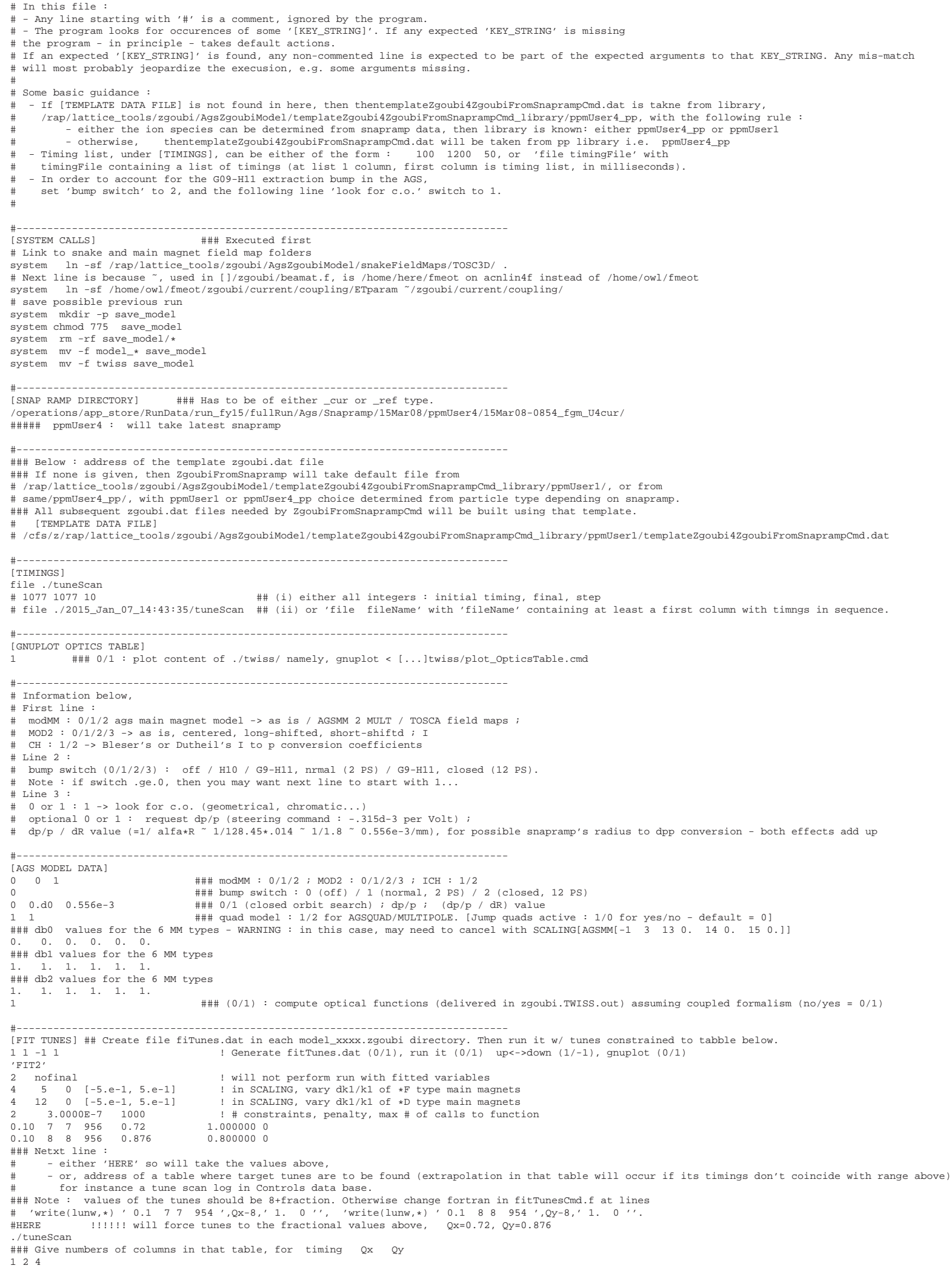




\section{D.3 zgoubi.dat}

AGS model in zgoubi: input data file to Zgoubi, taken from the archived example

/rap/lattice_tools/zgoubi/AgsZgoubiModel/AgsZgoubiModel_examples/AGSOptics/Example_2015 - 05 - 17
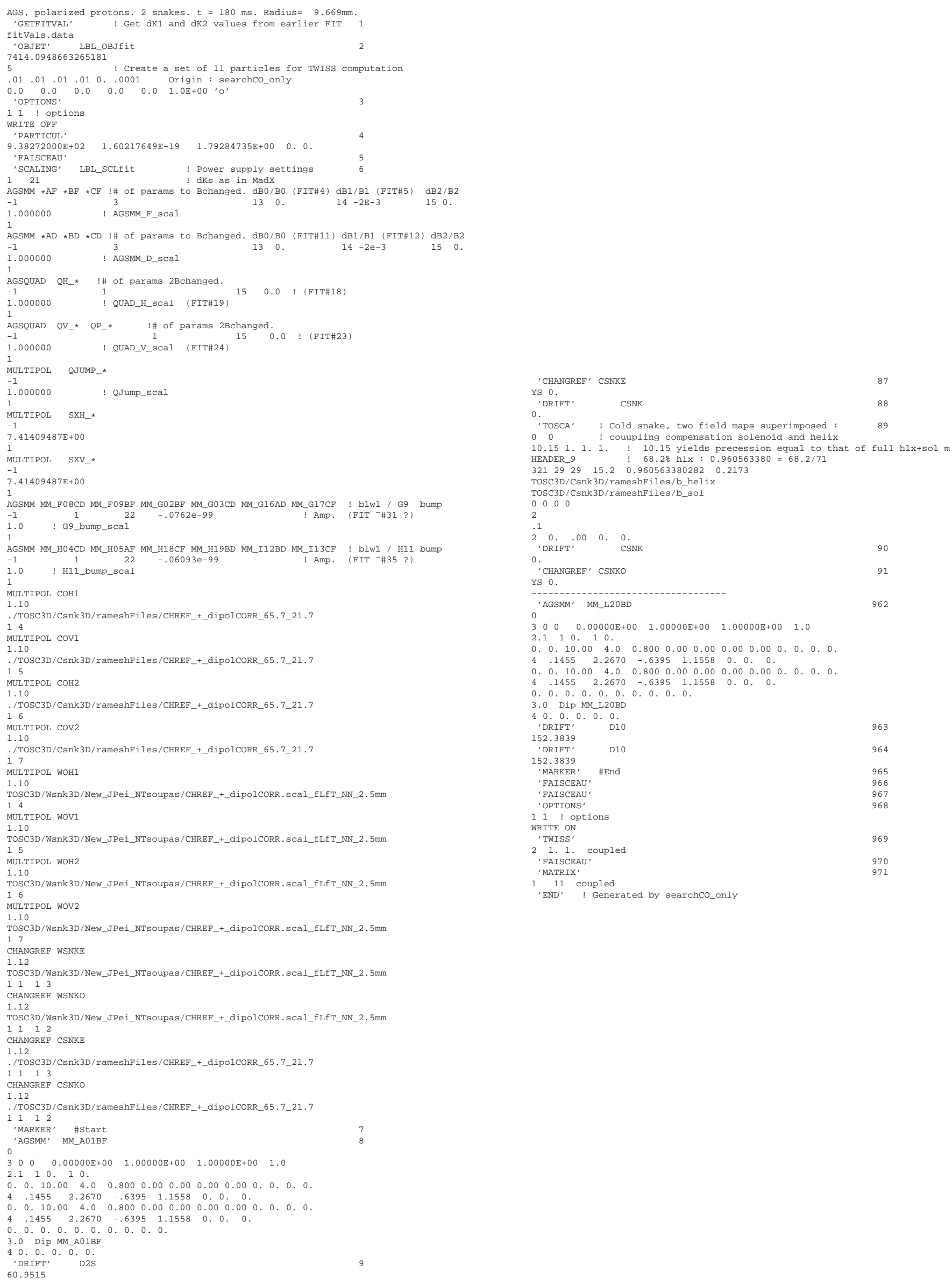


\section{D.4 twiss style output file}

Below are given specimen 'twiss' files out of Zgoubi model (left column) and of MADX (right). This is the example that yielded the data in Tab. 3, p.22.

\section{From Zgoubi}

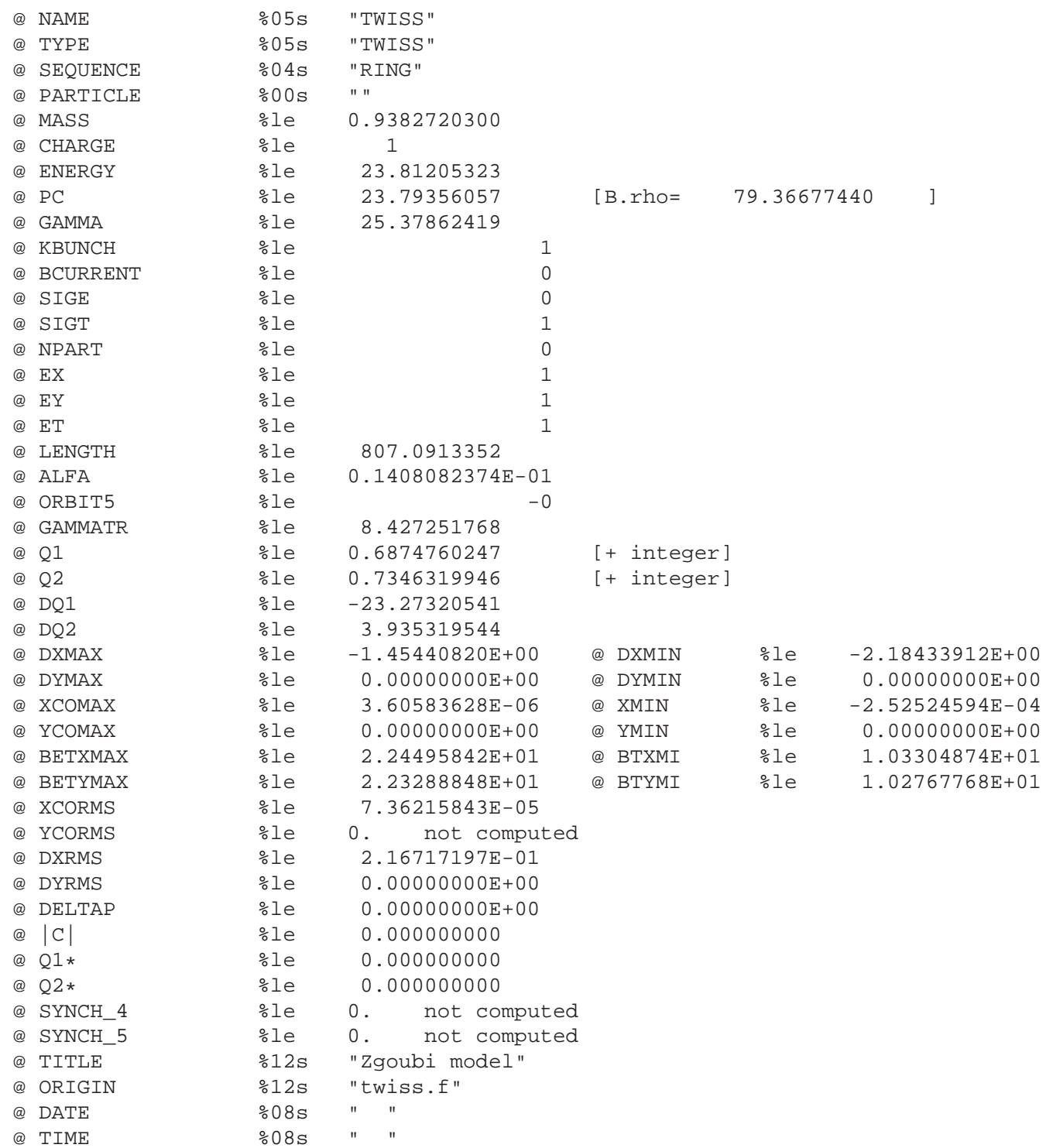

\section{From MADX}

a NAME

$05 \mathrm{~s}$ "TWISS"

a TYPE

$\% 05 s$ "TWISS"

a SEQUENCE

a PARTICLE

d MASS

a CHARGE

a ENERGY

a $\mathrm{PC}$

a GAMMA

a BCURRENT

a SIGE

a SIGT

a NPART

a $\mathrm{EX}$

c $E Y$

a $E T$

a LENGTH

a ALFA

a ORBIT5

a GAMMATR

c) Q1

a 22

a $\mathrm{DQ1}$

a $D Q 2$

a DXMAX

a DYMAX

e XCOMAX

a YCOMAX

C BETXMAX

a BETYMAX

a XCORMS

a YCORMS

a DXRMS

c DYRMS

a DELTAP

a SYNCH_1

a $\mathrm{SYNCH}-2$

d SYNCH_3

a $\mathrm{SYNCH}$

a SYNCH_5

a TITLE

e ORIGIN

e DATE

d TIME

$\circ 04 s$ "RING"
\%00s ""

\%le

ㅇe

ㅇe

le

le

$\circ 1 \mathrm{e}$
$\circ \mathrm{le}$

$\because 1 e$

$\% 1 e$

ㄱe

ㅇe

\%le

ole
$\% l e$
$\circ l e$
$\circ l e$

\%le

\% le

\%le

.

ole

$\circ l e$
$\vdots l e$
$\circ l e$

$\circ l e$
$\therefore l e$
$\circ l e$

ㅇe

ㄱe

일

ㄴe

\%le

ㄴe
0.93827203

23.81205303

23.79356037

$\begin{array}{ll}\text { \%le } & 23.79356037 \\ \text { \%le } & 25.37862397\end{array}$

\%le

$\div 35 \mathrm{~s}$ "AGS for Online Model (VHS, KAB, NM) 19s "MAD-X 3.04.14 Linux"

\%08s "03/06/16"

$\circ 08 \mathrm{~s} " 13.56 .49 "$ 


\section{References}

[1] The ray-tracing code Zgoubi, F. Méot, NIM A 767 (2014) 112-125.

[2] A numerical method for combined spin tracking and ray-tracing of charged particles, F. Méot, NIM A313 (1992) 492-500.

[3] Spin tracking simulations in AGS based on ray-tracing methods, F. Méot et al., BNL, Tech. Note C-A/AP/452 (Sept. 2009).

[4] Zgoubi-ing AGS : spin motion with snakes and jump-quads, $G \gamma=43.5 \longrightarrow G \gamma=46.5$ and beyond, F. Méot et al., BNL, Tech. Note C-A/AP/453 (Dec. 2009).

[5] Spin dynamics simulations at the AGS, H. Huang, W. W. MacKay, F. Méot, T. Roser, Procs IPAC 2010 Conf., Kyoto.

[6] Modelling of the AGS using Zgoubi - status, F. Méot et al., Procs IPAC 2011 Conf., San Sebastian.

[7] Development of a stepwise ray-tracing based, on-line model of the AGS, F. Méot et al., Procs PAC 2011 Conf., New York.

[8] Modelling of the AGS using Zgoubi - status, F. Méot et al., Procs PAC 2012 Conf., New Orleans.

[9] AGS On-Line Model Meeting Minutes, http://www.cadops.bnl.gov/AP/agsonlinemodel.htm

[10] Spin Meeting Minutes, http://www.cadops.bnl.gov/AP/spinmeeting.htm

[11] Y. Dutheil, Spin dynamics modeling in the AGS based on a stepwise ray-tracing method, PhD dissertation, BNL C-AD (2014).

[12] Where are the AGS snakes ?, F. Méot, R. Gupta, H. Huang, N. Tsoupas, BNL, Tech. Note C-A/AP/453 (May 2014).

[13] A RHIC-style IPM in the Brookhaven AGS.pdf, R. Connolly et al., BNL, Tech. Note C-A/AP/487 (2013).

[14] On the image in RHIC of AGS $\vec{n}_{0}$, via the AtR, F. Méot, H. Huang, N. Tsoupas, BNL, Tech. Note C-A/AP/502 (Jul 2013 ).

[15] On the Image of AGS ${ }^{3} \mathbf{H e}^{2+} \overrightarrow{\mathbf{n}}_{0}$, in the Blue, F. Méot, H. Huang, N. Tsoupas, BNL, Tech. Note C-A/AP/560 (Mar 2016).

[16] See accelerator conference publications by the present authors, from IPAC10 on.

[17] Acceleration of Polarized Protons in the AGS, N. Tsoupas et al., BNL, Tech. Note C-A/AP/391 (Feb 2010).

[18] Muon polarimeter in a neutrino factory decay ring, M. Apollonio et al., WEPE053, Procs. IPAC'10, Kyoto, Japan.

[19] 6-D beam dynamics simulations in FFAGs using the ray-tracing code Zgoubi, F. Méot, ICFA Beam Dyn.Newslett. 43:44-50 (2007).

[20] Spin code benchmarking at RHIC, F. Méot et al., Procs IPAC 2011 Conf., San Sebastian. Polarization transmission at RHIC, numerical simulations, F. Méot et al., Procs PAC 2012 Conf., New Orleans.

[21] On the effects of fringe fields in the LHC ring, F. Méot, Particle Accelerators, Vol. 52, 1996.

Undulator radiation simulation tools for LHC diagnostics, L. Ponce and F. Méot, Rep. CERN-SL-Note-2001-038 BI (2001).

[22] Improvement Plans for the RHIC/AGS On-Line Model Environments, K.A. Brown et al., http://accelconf.web.cern.ch/AccelConf/ICAP2009/papers/we3iodn03.pdf

[23] AGS Model in Zgoubi. RHIC Run 13 Polarization Modeling. Status, F. Méot at al., http://accelconf.web.cern.ch/AccelConf/IPAC2013/papers/wepea082.pdf

[24] RHIC injector complex on-line model status and plans, V. Schoefer et al., Proc. PAC09 Conf., Vancouver, 2009, http://accelconf.web.cern.ch/AccelConf/PAC2009/papers/fr5rep003.pdf

[25] Booster model in Zgoubi is under development by K. Hock, Operation Group, BNL C-AD.

[26] An online application to measure the dispersion function in AGS, N. Tsoupas et al., BNL C-A/AP/483, 5/10/2013.

[27] J. Chen et al., Cdev: An object-oriented class library for developing device control applications, Procs 1995 Int. Conf. on Accelerator and Large Experimental Physics Controls Systems, (Chicago), p. 97, 1995.

[28] Zgoubi Users' Guide, F. Méot, Tech. Note CA-AP/ (2012), Rep. BNL-98726-2012-IR. https://www.bnl.gov/isd/documents/79375.pdf

[29] Zgoubi on web, sourceforge site : https://sourceforge.net/projects/zgoubi/

[30] NERSC computing, on web : http://www.nersc.gov/

[31] Private communication, P. Adams, BNL C-AD, 2015. 
[32] F. Lin, Towards full preservation of polarization of proton beam in the AGS., Ph.D. Thesis, Indiana University (2007).

[33] Spin Dynamics Simulations and Horizontal Intrinsic Resonance Studies in the AGS using the Zgoubi Code, Y. Dutheil et al., MOPWA085, Procs IPAC 2013 Conf.

[34] Spin dynamics simulations and horizontal intrinsics resonance studies in the AGS using the Zgoubi code, Y. Dutheil et al., Procs NA-PAC 2013 Conf., Pasedena.

[35] V. Schoefer, private communication.

[36] F. Méot, V. Schoefer, to be published as a C-A/AP Tech. Note, draft manuscript with complete Zgoubi simulation results, including comparison with MAD, available at /rap/lattice_tools/zgoubi/AgsZgoubiModel/AgsZgoubiModel_examples/agsWithSurveyData/Log/log_surveyAGS.pdf

[37] Cold AGS Snake Optimization by Modeling, A.U. Luccio et al., BNL C-A/AP/128, Dec. 2003.

[38] N. Tsoupas et al., Closed orbit calculations at AGS and extraction beam parameters at H13, Tech. Note AD/RHIC/RD-75, BNL (Oct. 1994).

[39] Achieving Proton Polarization Goals for eRHIC, H. Huang et al., EIC'14 workshop, JLab (2014) https://www.jlab.org/conferences/eic2014/

[40] Accelerating Polarized He3 Beam in eRHIC, M. Bai et al., EIC'14 workshop, JLab (2014), https://www.jlab.org/conferences/eic2014/

[41] Transport of polarized ${ }^{3} \mathrm{He}$ in Booster and AGS, F. Méot, Workshop on Opportunities for Polarized He-3 in RHIC and EIC, BNL, Sept. 2011.

[42] V. Bargmann, L. Michel, V.L. Telegdi, Precession of the polarization of particles moving in a homogeneous electromagnetic field, Phys. Rev. Lett. 2 (1959) 435.

[43] The optimum central orbit in the AGS, A. Bleser, AGS Tech. Note TN 217, July 1985.

[44] Where are the AGS magnets ?, A. Bleser, AGS Tech. Note TN 215, May 20, 1985.

[45] The quadrupole and the sextupole fields of the AGS main magnets, R. Thern, E.J. Bleser, AGS/AD/Tech. Note No. 429 , March 4 , 1996. This document records, and parameterizes as a functin of momentum, the strength of the quadrupole (K1) and sextupole (K2) field in the AGS main magnets.

[46] The dipole fields of the AGS main magnets, R. Thern, E.J. Bleser, AGS/AD/Tech. Note No. 424, Jan. 26, 1996. This document records, and parameterizes as a function of momentum, the strength of the dipole field in the AGS main magnets (the 6 different types); correspondances between coil current, dipole field, proton momentun are tabulated.

[47] The parameters of the bare AGS, E.J. Bleser, AGS/AD/Tech. Note No. 430, March 15, 1996. This document recapitulates the properties of the bare AGS as calculated by MAD, and gives a parameterization as a function of momentum of tunes and chromaticities.

[48] C.J. Gardner, Notes on orbit equations in the AGS, Tech. Note C-A/AP/164, BNL C-AD (Sept. 2004).

[49] A Model of the AGS Based on Stepwise Ray-Tracing Through the Measured Field Maps of the Main Magnets, Y. Dutheil et al., Procs PAC 2012 Conf., New Orleans.

[50] MAD8 to Zgoubi, and MADX to Zgoubitranslators are available at /rap/lattice_tools/zgoubi/tools

[51] W. Meng, N. Tsoupas, private communication.

[52] See for instance : Design of a prototype gap shaping spiral dipole for a variable energy protontherapy FFAG, T. Planche et al., NIM-A 604 (2009) 435-442.

[53] Determination of KEK $150 \mathrm{MeV}$ FFAG parameters from ray-tracing in TOSCA field maps, F. Méot, M. Aiba, CERN NUFACT Note 140 (2003). 